\title{
The brain-specific Neural Zinc Finger transcription factor 2b (NZF-2b/7ZFMyt1) causes suppression of cocaine-induced locomotor activity
}

\author{
Vijay Chandrasekar, Jean-Luc Dreyer* \\ Division of Biochemistry, Department of Medicine, University of Fribourg, Rue du Musée 5, CH-1700 Fribourg, Switzerland
}

\begin{abstract}
Chronic cocaine induces high expression of the brain-specific Neural-Zinc-Finger transcription factor-2b (NZF-2b/7ZFMyt1), particularly in the mesolimbic dopaminergic pathway, resulting in a 11-fold increase in NZF-2b/7ZFMyt1 expression in the Nucleus Accumbens (NAc). Overexpression of this gene in the NAc with a NZF-2b/7ZFMyt1-expressing lentivirus resulted in $>55 \%$ decrease in locomotor activity upon chronic cocaine administration, compared to control animals. In contrast knocking-down the gene in the NAc with lentiviruses expressing shRNAs against NZF-2b/7ZFMyt1 induced strong hyperlocomotor activity upon cocaine. Strong inhibition of BDNF is observed upon NZF-2b/7ZFMyt1 expression, concomitant with strong induction of transcription factors REST1 (RE silencing transcription factor-1) and NAC1, probably leading to regulation of gene expression by interaction with histone deacetylases. These changes lead to decreased responsiveness of the animal to the locomotor-activating effects of cocaine, indicating that NZF-2b/7ZFMyt1 expression plays an important role in phenotypic changes induced by the drug.
\end{abstract}

\section{Introduction}

Drugs of abuse alter synaptic strength and induce long-term synaptic plasticity in the brain's reward system (Nestler, 2000). This constitutes the cellular mechanism that directly underlies addictive behaviors long after drug exposure (Conrad et al., 2008; Lüscher and Bellone, 2008). Identification of drug-induced alterations in gene expression and gene regulation is therefore key to understanding molecular adaptations underlying addiction. Drug-evoked plasticity has been reported in the ventral tegmental area (VTA), the nucleus accumbens (NAc) and the prefrontal cortex (Nestler, 2000). However only very few transcription factors (NAC1, CREB and delta-FosB) (Cha et al., 1997; Mackler et al., 2000; Kalivas et al., 1999; McClung et al., 2004) have been implicated as of today in gene regulation in the context of drug-induced plasticity. A further screening for transcription factors related to chronic cocaine administration revealed that a brain specific transcription factor, Neural zinc finger transcription factor $2 b$ (NZF-2b) may play a key role. NZF-2b, also designated as 7 zinc finger Myelin Transcription Factor-1 (7ZFMyt1), is expressed both

Abbreviations: CPU, caudate putamen; DA, Dopamine; GFP, green fluorescent protein; HEK293T, human embryonic kidney 293T cells; NAc, nucleus accumbens; qRTPCR, quantitative real-time polymerase chain reaction; shRNA, short hairpin RNA; siRNA, small interference RNA; VTA, ventral tegmental area; TFBSm, transcriptional factor binding site modules; BDNF, brain derived neurotrophic factor; REST, RE1silencing transcription factor 1; MECP2, methyl-cytosine binding protein; HDAC, histone deacetylase; BDNF, brain derived neurotrophic factor.

* Corresponding author. Fax: +410263009735.

E-mail address: jean-luc.dreyer@unifr.ch (J.-L. Dreyer). in neurons and also in oligodendrocytes, where it tightly regulates myelin-specific gene expression of proteolipid proteins PLP1, MAL2, MAG (Kim and Hudson, 1992; Strausberg et al., 2003; Nielsen et al., 2004). NZF-2b/7ZFMyt1 is a member of a neural-specific transcription factor gene family (Kim and Hudson, 1992; Strausberg et al., 2003), comprised of three Zn-finger genes [Myt1, Myt1L (Myt1-Like) and NZF3] that are expressed predominantly in the developing CNS. Both Myt1 and Myt1L proteins reside in distinct domains within the neuronal and oligodendrocyte nuclei, expressed as discrete patterns (Armstrong et al. 1995; Kim et al., 1997) and they interact with the corepressor Sin3B and actively recruit HDAC1 and HDAC2 to specific genes (Romm et al., 2005). The NZF-2/Myt1 comprises two isoforms, the poorly expressed NZF-2a/Myt1 (6-zinc finger form) and the predominant NZF-2b/7ZFMyt1 (7-zinc-finger form), located on chromosome 20 and 2 of human and mouse, respectively (Nagase et al., 1998; Kikuno et al., 1999; Matsushita et al., 2002; Deloukas et al., 2002; Nielsen et al., 2004). But the role of these two isoforms in neurons is poorly known.

In the present study we show that cocaine administration induces high expression of NZF-2b/7ZFMyt1 particularly in the mesolimbic dopaminergic pathway and that its overexpression in the NAc results in significant hypolocomotor activity upon cocaine administration, whereas NZF-2b/7ZFMyt1 knock-down induces hyperlocomotor activity. Furthermore, increased expression of NZF-2b/7ZFMyt1 also induces differential expression of other genes, possibly resulting in regulation of downstream genes by epigenetic modification. Together these data points to the relevant role of NZF-2b/7ZFMyt1 in regulatory mechanisms of genes related to chronic cocaine administration. 


\section{Experimental procedures}

Animal handling

All animal experiments were carried out in accordance with guidelines and regulations for Animal Experimentation, BAG, Bern, Switzerland. Male Wistar rats weighing 250-300 g (BRL, Fillingsdorf, Switzerland) were used for all the experiments. The animals were housed in groups (four per cage) in clear plastic cages with wire grid lids. The animals were kept on a 12-h light/dark cycle (lights off at $07.00 \mathrm{~h}$ ), with access to food and water ad libitum. For doxycycline treatment, animals were maintained with $0.02 \%$ doxycycline (Sigma) and 5\% sucrose supplemented in water. During the gene overexpression period, animals were maintained only with $5 \%$ sucrose.

\section{Computational promoter analysis}

Promoter analysis was performed by computational methods using softwares available in the public domain as well as promoter analysis tools from Transfac (http://transfac.gbf.de/) and Genomatix (http://www.genomatix.de/). Genomic sequences 3000 bps upstream of the transcription start site of a large set of selected genes were retrieved from Eukaryotic Promoter database (http://www.epd.isbsib.ch/) and the highest scoring putative functional transcription factor binding site modules (TFBSm) were chosen based on stringent orthologous species conservation constraints. Genes were chosen based on evident implication in cocaine administration by literature knowledge. For the computational prediction of MYT1 binding sites in target gene promoters, the software utilizes the MYT1 matrix family with an optimized matrix threshold of 0.75 and maintenance of at least the less stringent requirement of AAGTT motifs (Kim and Hudson, 1992) to predict a more relevant match. MYT1 matrix has a re-value of $<0.01$, i.e., a random expectation of such a binding site is less than $<0.01$ matches per $1000 \mathrm{bp}$. As with most transcription factors (excluding TATA-box, etc.), MYT1 matrix recognizes symmetric or palindromic sites and have binding sites in both orientations in promoters or enhancers with a consensus DNA binding site sequence of at least two AAA(G/C)TTT motifs (Bellefroid et al., 1996).

\section{NZF-2b/7ZFMYT1 lentiviral expression constructs}

The lentiviral expression construct of the myelin transcription factor-1 (NZF-2b/7ZFMYT1) gene was generated from the 7ZFMYT1 plasmid constructs kindly provided by Prof. Armstrong's lab (Uniformed Services University of the Health Sciences, Bethesda). The 7 zinc finger MYT1 gene coding region along with the Myc tag ( 3.9 kb) from pMycMyt1-7zf-IRES/RED expression vector was first digested with EcoR I and sub-cloned into pDRIVE vector (QIAGEN). 7ZFMYT1 gene ORF was then digested with SnaB I and Xho I from pDRIVE and cloned into the lentiviral vector pTK-431 using Hpa I and Xho I sites that express the gene of interest under control of a Tet-Off promotor. The clones were verified by conventional methods. The green fluorescent protein (GFP) expressing lentiviral vector pTK433 (Bahi et al., 2004; Boyer and Dreyer, 2007) was used as a control vector.

\section{ZFMyt1-siRNA lentiviral expression constructs}

Three different siRNAs targeting different regions of the NZF-2b mRNA sequence were constructed to silence NZF-2b/7ZFMyt1 expression in vitro and in vivo. The following targets within the NZF-2b mRNA sequence were selected, based on Hannon's design criterion (http://katahdin.cshl.org:9331/RNAi/html/rnai.html): first target: bp 1127-1145; second target: bp 2239-2268 and third target: bp 3487-3507. To each oligonucleotide, a Xho I restriction site was added at $3^{\prime}$ and a U6-3'-specific 10 mer at $5^{\prime}$. Using the pSilencer 1.0 U6 (Ambion, UK) as a template and a U6 promoter-specific forward primer containing BamHI restriction site (5'-GCG GAT CCC GCT CTA GAA CTA GTG C-3'), each siRNA target was added to the mouse U6 promoter by PCR, using the following PCR program: 120 s at $93{ }^{\circ} \mathrm{C}$ (initial denaturation) followed by 35 cycles $\left(45 \mathrm{~s}\right.$ at $92^{\circ} \mathrm{C}, 45 \mathrm{~s}$ at $64{ }^{\circ} \mathrm{C}$ and $45 \mathrm{~s}$ at $72{ }^{\circ} \mathrm{C}$ ) in $5 \%$ dimethyl sulphoxide (Sigma, Switzerland). The PCR product was directly ligated into pDRIVE vector from QIAGEN PCR Cloning Kit (QIAGEN). The shRNA insert along with the U6 promoter was then digested with BamHI and Xho I, sub-cloned into similar sites in the lentiviral vector pTK-431, and sequenced to verify the integrity of each construct.

\section{Lentivirus production}

The lentiviral vector expression plasmids (either pTK4317ZFMyt1, pTK433-GFP or pTK431-U6-7ZFMyt1-siRNAs), along with the packaging construct plasmid $\mathrm{p} \Delta \mathrm{NRF}$ and the envelope plasmid pMDG-VSV-G were co-transfected into human embryonic kidney (HEK) 293T cells to produce the viral particles (Bahi et al., 2004; Boyer and Dreyer, 2007). The viral titers were determined by p24 antigen measurements (KPL, USA). For the in vivo experiments, the different viral stocks were matched for viral particle content and used at $0.2 \mathrm{mg} / \mathrm{mL}$ of $\mathrm{p} 24$.

\section{Cocaine administration}

Rats $(n=8)$ were given single daily i.p. injection of $15 \mathrm{mg} / \mathrm{kg}$ cocaine- $\mathrm{HCl}$ for a period of 15 days. Control animals received $0.9 \%$ saline i.p. injections under the same schedule (Bahi et al., 2004; Boyer and Dreyer, 2007). All animals were sacrificed by decapitation $24 \mathrm{~h}$ after the last injection and the brains were removed and stored rapidly in TRIzol reagent (Invitrogen).

\section{Stereotaxic surgery}

Stereotaxic surgery and lentiviral injection were performed according to previous publications (Bahi et al., 2004; Boyer and Dreyer, 2007). Rats were anesthetized with ketamine-xylazine $(10 \mathrm{mg} / \mathrm{kg}$ and $0.1 \mathrm{mg} / \mathrm{kg}$ ) administered i.p. Animals were bilaterally injected into the NAc with $2 \mu \mathrm{L}$ of concentrated lentiviral expression system stock $\left(0.2 \mathrm{mg} / \mathrm{mL}\right.$ of $\mathrm{p} 24$, corresponding to $\left.8 \times 10^{9} \mathrm{IU} / \mathrm{mL}\right)$. The injections were performed bilaterally at the following coordinates, as calculated from bregma and the dura mera: anterior +1.4 ; lateral \pm 1.2; ventral -6.8 (Paxinos \& Watson, 1998).

\section{Open field locomotor activity behavioral experiment}

Four groups of animals ( $n=9$ ) were used for the open field cocaine induced locomotor activity monitoring experiment. The first group was stereotaxically injected with $2 \mu \mathrm{L}$ of Lenti-GFP (controls); the second group was injected with $2 \mu \mathrm{L}$ of Lenti-7ZFMyt1; the third group was injected with $2 \mu \mathrm{L}$ of a mix of all three Lenti-7ZFMyt1siRNAs; and the fourth group was injected with $2 \mu \mathrm{L}$ Lenti7ZFMyt1together with $2 \mu \mathrm{L}$ of a mix of the three Lenti-7ZFMyt1siRNAs. 7-12 days after stereotaxic surgery, locomotor activity was monitored in daily sessions in $43.2 \times 43.2 \mathrm{~cm}$ MED-OFA-RS cages (MED Associates, USA) during the dark cycle, according to previously published procedures (Bahi et al., 2004; Boyer and Dreyer, 2007). Animals were injected daily with cocaine i.p. and were fed without doxycycline for 5 days (Session A), then fed doxycycline for 7 days (Session B), and finally fed without doxycycline for 7 days (Session C). Locomotor activity was recorded on days 1-5, 8-12 and 15-19; over the 2-day periods between sessions - necessary for full doxycyclinemediated switch of ectopic NZF-2b/7ZFMyt1expression - no measurements were performed (Table 1 ). Prior to each locomotor activity 
Table 1

Experimental protocol for each group of animals used in open field locomotor activity experiment.

\begin{tabular}{|c|c|c|c|c|c|c|c|c|c|c|c|c|c|c|c|c|c|c|c|c|c|c|c|c|}
\hline Days & 1 & 2 & 3 & 4 & 5 & 6 & 7 & 8 & 9 & 10 & 11 & 12 & 13 & 14 & 15 & 16 & 17 & 18 & 19 & 20 & 21 & 22 & 23 & 24 \\
\hline Sessions & A & & & & & & & B & & & & & Sw & & C & & & & & Switch or S & rifice & & & \\
\hline Doxycycline treatment & - & - & - & - & - & + & + & + & + & + & + & + & - & - & - & - & - & - & - & - & + & + & + & + \\
\hline $\begin{array}{l}\text { Activity measurement } \\
\text { Sacrifice }\end{array}$ & $\sqrt{ }$ & $\sqrt{ }$ & $\sqrt{ }$ & $\sqrt{ }$ & $\sqrt{ }$ & $\times$ & $x$ & $\sqrt{ }$ & $\sqrt{ }$ & $\sqrt{ }$ & $\sqrt{ }$ & $\sqrt{ }$ & $x$ & $x$ & $\sqrt{ }$ & $\sqrt{ }$ & $\sqrt{ }$ & $\sqrt{ }$ & $\sqrt{ }$ & $\begin{array}{l}\times \\
n=5 \text { of } \\
\text { each group }\end{array}$ & $\times$ & $x$ & $x$ & $\begin{array}{l}\times \\
\text { rest }(n=4) \\
\text { of each group }\end{array}$ \\
\hline
\end{tabular}

At the end of the experiment animals are sacrificed and brain tissues were utilized for immunohistochemistry or qRT-PCR (see Materials and methods).

measurements, the animal was injected with $0.9 \%$ saline, and placed into the activity-monitoring cage for a 15 -min baseline measurement. After this 15 -min period, the session automatically paused for a few seconds and, during this interval, each subject received cocaine- $\mathrm{HCl}$ (Sigma, $15 \mathrm{mg} / \mathrm{kg}$, i.p.) and was then placed back into the locomotor activity-monitoring cage for a further $60 \mathrm{~min}$. Statistical evaluation of behavioral analysis was performed according to previous publications (see Bahi et al., 2004; Boyer and Dreyer, 2007). At the end of the third session (Session $\mathrm{C}$ ) half of the animals were sacrificed (Table 1 ). The other half were switched back on doxycycline for $48 \mathrm{~h}$ then killed. All animals were killed by decapitation. During this switch period, animals were given continuous daily administration of cocaine. All groups were sacrificed $24 \mathrm{~h}$ after the last cocaine injection. Brains were used for either immunohistochemistry or quantitative real-time PCR.

\section{Total RNA isolation}

Rat brain parts, micro-dissected from cocaine and saline treated animals, or HEK-293 cells infected with lentivirus for gene expression were homogenized in TRIzol reagent (Invitrogen), followed by RNA isolation using chloroform extraction and isopropanol precipitation. After being washed with $70 \%$ and $100 \%(\mathrm{v} / \mathrm{v})$ ethanol, RNA pellets were dissolved in DEPC-treated $\mathrm{H}_{2} \mathrm{O}$. Quality and integrity of the RNA was analyzed and quantified accurately by a Bioanalyzer and $2 \%$ agarose gel. All RNA samples were stored at $-70{ }^{\circ} \mathrm{C}$.

\section{NZF-2b/7ZFMYT1 transcript quantification by real-time quantitative $P C R$}

RNA from cocaine and saline-treated animal brain parts were reverse transcribed using M-MLV Reverse Transcriptase (Promega). $2 \mu \mathrm{g}$ of RNA from each sample was reverse transcribed at $42{ }^{\circ} \mathrm{C}$ for 30 min with $1 \mu$ g of oligo(dT) or specific primers, 5 first strand buffer, $100 \mathrm{mM}$ DTT, $10 \mathrm{mM}$ dNTP, RNAsin (Invitrogen) and M-MLV. cDNAs were checked for their optimum dilution in subsequent real-time PCR reactions. Primer sets for rat NZF-2b/7ZFMyt1, $\beta$-actin or cyclophilin were designed to amplify 100-200 bp products, using PRIMER3 software: (http://frodo.wi.mit.edu/cgi-bin/primer3/primer3_www.cgi). PCR reaction mixtures included cDNAs in optimum dilution, the SYBR-Green qPCR Master mix (BioRad, Reinach, Switzerland), $10 \mu \mathrm{M}$ primers, in a total reaction volume of $20 \mu$ l. Expression profiling was done with dissociation curves using iCycler (BioRad, Reinach, Switzerland). Cycling parameters were $95{ }^{\circ} \mathrm{C}$ for 4 min followed by 40 cycles of $20{ }^{\circ} \mathrm{C} / \mathrm{s}$ temperature transition rate up to $95{ }^{\circ} \mathrm{C}(30 \mathrm{~s}), 62{ }^{\circ} \mathrm{C}(45 \mathrm{~s})$, followed by melting curve analysis. All reactions were performed in triplicates, with reference dye normalization ( $\beta$-actin or cyclophilin RNA), and the median $C_{t}$ (cycle threshold) value was used for analysis. To determine the linearity and detection limit of the assay, cDNA samples were amplified for successive 10-fold dilutions in a series of real-time PCRs, using a duplicate assay on each dilution, so that the correlation coefficient could be calculated from the standard curve of $C_{t}$ values. Comparisons were made between cocaine and saline groups. Significance was calculated using two-way ANOVA followed by Bonferroni post hoc tests and the level of statistical significance was set at $P<0.001$. Data were expressed as means \pm SEM. The $\Delta C_{t}$ for each candidate was calculated as: $\Delta C_{\mathrm{t}}=\left[C_{\mathrm{t}}\right.$ (candidate) $-C_{\mathrm{t}}$ (cyclophilin or $\beta$-actin $\left.)\right]$. The relative abundance of each target can be calculated as the ratio between treated and untreated samples (Bahi et al., 2004; Boyer and Dreyer, 2007). The PCR reaction was evaluated by melting curve analysis and by checking the PCR products on $2 \%$ agarose gel.

The following primer sets were used for amplification: 7ZFMYT1: forward 5'-GCA GAC CTC AGT TGT CCT ACC-3' and reverse 5'-CTT GGA TAC CAG GTG CTC AG-3'; cyclophilin: forward 5'-GTG AGA AGG GCT TTG GCT AC-3' and reverse 5'-TTC TCG TCA GGA AAG CGG-3'; $\beta$-actin: forward 5'-AGC CAT GTA CGT AGC CAT CC-3' and reverse $5^{\prime}$-CTC TCA GCT GTG GTG GTG AA-3'; BDNF: forward 5'-GGT TCG AGA GGT CTG ACG AC-3' and reverse 5'-CAA AGG CAC TTG ACT GCT GA-3'; REST1: forward 5'-CGA GTT GAT GCC TGT TGG AGA C-3' and reverse 5'-TGC TTC AAA TAC GGG CTG GG-3'; NAC1: forward 5'-GCT CTT CCT GAG CAG GTC GT-3' and reverse 5'-GTG CCT GTC ACA AGC TCC AG-3'.

\section{Immunohistochemistry}

To check the expression of NZF-2b/7ZFMyt1 after lentiviral injections, rats were decapitated, brains were quickly removed, immediately frozen in isopentane (at $-30{ }^{\circ} \mathrm{C}$ for $3 \mathrm{~min}$ ) and kept at $-25^{\circ} \mathrm{C}$. Coronal sections were cut at $14 \mu \mathrm{m}$ in a cryostat and placed on gelatinized glass slides, air-dried at room temperature for $20 \mathrm{~min}$ and kept at $-25^{\circ} \mathrm{C}$ until further processing.

Brain sections were fixed for $10 \mathrm{~min}$ in cold acetone and washed three times in $1 \times$ phosphate-buffered saline (PBS). Non-specific binding sites were blocked by incubating slices for $1 \mathrm{~h}$ in $1 \times \mathrm{PBS}$ containing $1 \%$ bovine serum albumin, $1 \%$ Triton X-100 and $3 \%$ normal goat serum. Sections were then incubated overnight at $4{ }^{\circ} \mathrm{C}$ with primary antibodies specifically against either endogenous NZF-2b/ 7ZFMyt1 (with rabbit polyclonal anti-MYT1 antibody; 1:15; Sigma prestige antibodies HPA006303) or against Myc for ectopic Myctagged protein (using either mouse monoclonal anti-Myc tag antibody, Sigma M5546, 1:200 or rabbit polyclonal anti-Myc-tag antibody; 1:1000; Abcam ab9106) or against NeuN (with mouse monoclonal anti-neuronal-nuclei, MAB 377, Chemicon, 1:5000) or against GFAP (anti-glial fibrillary acidic protein (astrocyte marker), Abcam, ab10062, 1:1000). Antibodies were diluted in $1 \times$ PBS containing $0.1 \%$ Triton $\mathrm{X}-100$ and $1 \%$ normal goat serum. Thereafter, slices were washed three times in $1 \times \mathrm{PBS}$, and incubated for $2 \mathrm{~h}$ in the secondary antibody (either Texas red-conjugated goat anti-rabbit immunoglobulin G, 1:5000; or FITC-conjugated goat anti-mouse IgG, 1:5000; or HRP-conjugated goat anti-rabbit, $1: 5000)$ in $1 \times$ PBS containing $0.1 \%$ Triton $\mathrm{X}-100$. The sections were coverslipped with a medium containing glycerol in PBS (AF1 mounting solution, Citifluor). For double staining experiment with NeuN, the slices were first treated with anti-Myc or anti-MYT1 primary and secondary antibody followed by treatment with NeuN primary and secondary antibody. For double staining experiments with Hoechst 33342 (1:1500, Sigma, Steinheim, Germany) nuclei were stained by 30 -min incubation. Negative controls included omission or substitution of primary antibodies. 
Fluorescence microscopy was performed to observe the stained sections using a multifluorescence microscope (Axioplan 2 imaging; Zeiss). Fluorophores used (FITC and Texas red) were detected with the appropriate detecting systems (HAL 100). FITC was excited at $495 \mathrm{~nm}$ and was detected through a light path ranging 510-550 nm. Texas red was exited at $570 \mathrm{~nm}$ and was detected through a light path ranging 600-660 $\mathrm{nm}$. Stained sections were visualized, photographed using a multichannel camera (Axiocam, Zeiss) combined with acquisition software (Axiovision system 3.1) and recorded on CD (Boyer and Dreyer, 2007).

\section{Statistical analysis}

For chronic expression profile of 7ZFMyt1, statistical analysis was done using two-way ANOVA and Student's paired one-tailed t-test to compare between saline and cocaine treated samples. Two-way ANOVA was used to compare both lentiviral and drug treatment in the presence or absence of doxycycline, using GraphPad PRISM (V3.0, GraphPad, San Diego, CA). For locomotor activity two-way ANOVA was used, with time as the within-subject factors and lentiviral gene expression as the between-subject factors followed by Bonferroni post hoc tests. Quantitative real-time PCR results were analyzed by two-way ANOVA, with drug treatment (water vs. doxycycline) as the within-subject factors and lentiviral-mediated gene expression as the between-subject factors followed by Bonferroni post hoc tests.

\section{Results}

In silico promoter analysis of cocaine modulated genes to identify putative cocaine responsive transcription factor

In this study, we used in silico promoter analysis of a set of genes differentially expressed upon cocaine administration to identify potential candidate transcription factors involved in cocaine administration (Table S1 and Fig. S1, supplementary material). The results revealed the potential role of the brain specific transcription factor NZF-2b/7ZFMyt1. We have selected a large set of contextoriented genes, based on extensive literature search implicating them on cocaine administration pathway. Computational promoter analysis of these genes (horizontal gene selection) over a span of $>3000$ bp up stream of the transcriptional start site (data not shown) enabled to identify several transcription factors having putative functional transcriptional factor binding site modules (TFBSm) in several of the chosen genes. Although the occurrence of a single transcription factor binding site found by the software alone does not imply it is functional, functionality is determined by the sequence context. If a binding site is part of a framework of two or more sites there is strong evidence that the individual sites may be functional. Candidates for further functional module analysis were selected based on stringent conservation parameters, i.e., conservation of TFBSm among different genes involved in the same pathway and across orthologous species (e.g., human, rat, mouse). For the Myt1 binding site prediction in the gene promoters spanning $>3000$ bp upstream of the transcription start site, the known consensus binding site motif AAA(G/C)TTT (Kim and Hudson, 1992; Bellefroid et al., 1996) were utilized on both strands of the promoter and enhancer sequences. Next we checked the transcription factors with the highest scoring conserved functional modules (predicted) for specific expression patterns in the brain, by literature search and by available microarray data results. Further validation of the implicated transcription factors from the promoter analysis was done by quantitative RT-PCR. From our promoter analysis we found that NZF-2b/7ZFMyt1 has a highly conserved putative TFBSm in many of the genes analyzed (Table S1, Fig. S1). The fact that NZF-2b/ 7ZFMyt 1 is a brain and developmental stage specific transcription factor gave an indication that NZF-2b/7ZFMyt1 might have a significant role in the regulation of these established cocaine responsive genes.

Cocaine induces differential expression of the brain specific transcription factor NZF-2b/7ZFMyt1 (Neural-Zinc-Finger transcription factor 2b/7ZF-Myelin transcription factor 1) in different brain regions

To assess the function of NZF2b/7ZFMyt1 in cocaine administration, a group of animals $(n=8)$ was injected daily $15 \mathrm{mg} / \mathrm{kg}$ of cocaine i.p. over a period of 15 days, and a control group was injected $0.9 \%$ saline. Thereafter animals were sacrificed by decapitation and brains were micro-dissected for RNA isolation to be used for qRT-PCR. Primers specific to the 7zf-Myt1 (Nielsen et al., 2004) were used and were normalized against cyclophilin. The results show that 7zf-Myt1 expression was significantly increased following chronic cocaine administration (Fig. $1 ; F_{1,24}=971.91, P<0.0001$ ). A 11-fold upregulation of NZF-2b/7ZFMyt1 was observed in the nucleus accumbens (NAc) $(P<0.0001)$, compared to the saline treated control group. This was correlated with a two-fold up-regulation in the ventral tegmental area (VTA) $(P<0.05)$, a very strong induction of about 30 fold increase in the hippocampus (HIP) $(P<0.0001)$, compared to negligible basal level expression, and 2.4-fold increase in the prefrontal cortex (PFC) $(P<0.05)$. A slight, but not significant downregulation was found in the caudate putamen. Other brain regions analysed ("rest of the brain," ROB) $(P<0.0001)$, displayed a 5.5-fold increase in NZF-2b/7ZFMyt1 expression (Fig. $1 ; F_{5,24}=100.38$, $P<0.0001$ ). These data correlate with in silico results, which showed that NZF-2b/7ZFMyt1 displays highly conserved TF binding modules in many cocaine-modulated genes and thus may be differentially expressed upon cocaine administration.

Furthermore, a group of animals $(n=8)$ was injected with four high doses of cocaine ( $30 \mathrm{mg} / \mathrm{kg}$ i.p.) at 2-h interval within a single day whereas a control group $(n=8)$ received $0.9 \%$ saline under the same schedule that mimics the drug abuse in humans and allows for easy assessment of neurobiological changes associated with addiction (Michna et al., 2002). Quantitative RT-PCR revealed that significant increase in gene expression of NZF-2b/7ZFMyt1 was observed in the NAc and in the Hip but not in other brain regions examined (data not shown). Together the NZF-2b/7ZFMyt1 expression profile in these two different cocaine administration paradigms showing a strong induction in the expression of NZF-2b/7ZFMyt1 in specific brain regions related to the mesolimbic dopaminergic pathway supports our hypothesis on its role in gene regulation upon cocaine exposure.

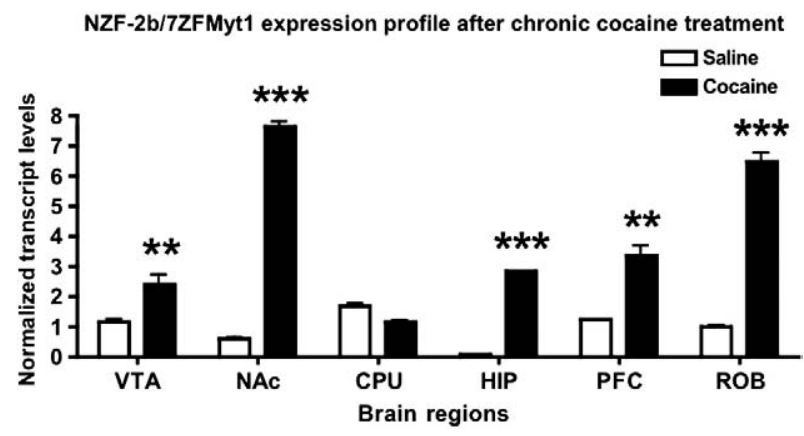

Fig. 1. Chronic cocaine administration induces differential expression of the brainspecific transcription factor NZF-2b/7ZFMyt1 (Neural-Zinc-Finger transcription factor 2b/7ZF Myelin transcription factor 1) in different brain regions. Quantification of NZF2b/7ZFMyt1 mRNA levels by qRT-PCR from rats $(n=8)$ after either chronic cocaine administration $(15 \mathrm{mg} / \mathrm{kg} /$ day) or saline (control) (see Methods for details). Expression levels were calculated relative to the endogenous housekeeping gene cyclophilin. ${ }^{*} P<0.05$; ${ }^{* *} P<0.01$; ${ }^{* * *} P<0.001$ by Student's paired $t$-test, and compared to saline treated samples. VTA: ventral tegmental area; NAc: nucleus accumbens; CPU: caudate putamen; HIP: hippocampus; PFC: prefrontal cortex; ROB: rest of the brains. 
NZF-2b/7ZFMyt1 expression in the NAc decreases locomotor activity upon cocaine administration

To further reveal the implications of NZF-2b/7ZFMyt1 gene expression in behavior, we constructed a doxycycline-regulatable lentiviral vector, Lenti-7ZFMyt1, holding the mouse NZF-2b/ 7ZFMyt1 cDNA, as well as three non-regulatable lentiviral vectors, Lenti-7ZFMyt1-siRNA1, Lenti-7ZFMyt1-siRNA2 and Lenti-7ZFMyt1siRNA3. The latter are expressing small hairpin RNAs, aimed at silencing NZF-2b/7ZFMyt1 expression and are specifically targeted against different regions of the NZF-2b/7ZFMyt1 mRNA (Materials and methods). In vitro studies confirmed that Lenti-7ZFMyt1 expressed NZF-2b/7ZFMyt1 and that the expression could be blocked efficiently and specifically with Lenti-7ZFMyt1-Sils (data not shown). These viruses were stereotaxically injected into the shell part of the NAc and the effects of their expression upon passive cocaine delivery on locomotor activity were assessed. Of the four groups of animals $(n=9)$, one group was injected with the doxycycline-regulatable Lenti-7ZFMyt1, resulting in local overexpression of the gene in absence of doxycycline; a second group was injected with Lenti-7ZFMyt1 together with a mix of three Lenti7ZFMyt1-siRNAs; a third group was injected only with a mix of three Lenti-7ZFMyt1-siRNAs, inducing local silencing of the gene; the fourth group was a control group injected with Lenti-GFP (green fluorescent protein). Behavioral evaluation was performed over a period of three weeks in three 5-day sessions: session $A$, no doxycycline; session $B$, animals fed doxycycline; then session $C$, no doxycycline, recapitulating both local gene expression of session $\mathrm{A}$ and initially observed behavioral manifestations. Chronic cocaine administration was continued daily throughout each sessions A, B and C. Between each session, a 2-day period without behavioral measurement was managed to enable full change in gene expression, during which animals were also daily injected cocaine. This protocol enables an accurate behavioral evaluation of the effects of local gene expression changes on the very same animals (Bahi et al. 2004, 2005a; Bahi and Dreyer, 2005).

Manipulations in the NZF-2b/7ZFMyt1 ectopic expression levels induced changes in the locomotor stimulant effects of cocaine as compared to control, GFP expressing group (Figs. 2A and B). When animals were injected with Lenti-GFP (control), a typical cocaine induced locomotor activity was observed, that remained unchanged when animals were fed doxycline (Figs. 2A and B). This was not surprising as previous work from our lab has shown that GFP overexpression in the NAc does not affect behavior compared to naive animals in cocaine induced locomotor activity (Bahi et al., 2004; 2005a; Bahi and Dreyer, 2005; Boyer and Dreyer, 2007). When animals were injected with Lenti-7ZFMyt1, 50-55\% reduction in the locomotor activity was observed (Figs. $2 \mathrm{~A}$ and $\mathrm{B}, F_{3,240}=218.96$, $P<0.0001$ ) under conditions of gene expression, i.e., in the absence of doxycycline $\left(F_{14,240}=120.30, P<0.0001\right)$. When these animals were fed doxycycline (session $B$ ), inducing down-regulation of ectopic NZF2b/7ZFMyt1 expression $\left(F_{14,240}=200.32, P<0.0001\right)$, locomotor activity was not significantly different from the GFP control group $\left(F_{3,240}=58.92, P<0.0001\right)$ under similar conditions; but removing doxycycline (session $C$ ) restored again the behavior observed during session A (Fig. 2). The other group of animals, injected with Lenti7ZFMyt1-siRNAs - inducing local suppression of endogenous NZF-2b/ 7ZFMyt1 expression - displayed a strong (50\%) increase of locomotor activity compared to GFP controls during session A, i.e., in absence of doxycycline $\left(F_{3,240}=218.96, P<0.0001\right)$, when fed doxycycline, no significant behavioral change was observed in these same animals compared to controls $\left(F_{3,240}=58.92, P<0.0001\right)$ (as expected, because Lenti-7ZFMyt1-siRNAs are not doxycycline-regulatable), and the same behavior was maintained by removing doxycycline in session $C\left(F_{3,240}=270.22, P<0.0001\right)$. Finally the group of animals injected with Lenti-7ZFMyt1 and a mix of three Lenti-7ZFMyt1-
siRNAs - displayed locomotor activity almost comparable to the GFP control group in sessions $\mathrm{A}$ and $\mathrm{C}$ (without doxycycline; $F_{3,240}=$ 218.96, $P<0.0001$ and $F_{3,240}=270.22, P<0.0001$, respectively), except for the shoulder regions of the curves: NZF-2b/7ZFMyt1 expression causes immediate response to cocaine injection, manifesting its peak in locomotor activity significantly earlier than the control GFP group. Upon doxycyline treatment (session B) that inhibits both ectopic and endogenous NZF-2b/7ZFMyt1 expression, these same animals displayed a $25 \%$ increase in locomotor activity compared to GFP group $\left(F_{3,240}=218.96, P<0.0001\right)$ (Figs. $2 \mathrm{~A}$ and $\left.\mathrm{B}\right)$.

Cocaine-induced behavioral sensitization was well observed for all groups during session A over the first 5 days (Fig. 2C; $F_{4,40}=5.57$, $P=0.0012$ ). The sensitization was significant in the Lenti-GFP and Lenti-Myt1 + Lenti-Myt1-siRNAs groups displaying a $40.5 \%$ and $~ 50 \%$ increase in locomotor activity over this session compared to $16 \%$ increase in Lenti-Myt1-siRNAs group. Sensitization effect observed in animals injected with Lenti-Myt 1 in the first 5 days of cocaine administration $\left(F_{3,40}=82.85, P<0.0001\right)$ were absent in sessions $\mathrm{B}$ and $C$. In the rest of the groups, during subsequent session B, only a modest non-significant level of sensitization was observed which was completely absent in the final session C.

\section{Quantification of NZF-2b/7ZFMyt1 mRNA levels after in vivo} Lenti-7ZFMyt1 expression in the NAC

To assess whether the behavioral changes observed were related to NZF-2b/7ZFMyt1 gene expression, animals were sacrificed after the behavioral experiments and NZF-2b/7ZFMyt1 mRNA levels were tested in the NAc and VTA by qRT-PCR. Lentiviruses have been injected in the NAc in all animals and mRNA from either the NAC or the VTA was isolated at the end of the behavioral experiments (as described in the previous section). As shown in Fig. 3A, animals injected Lenti-7ZFMyt1 displayed a 4-fold increase in expression of NZF-2b/7ZFMyt1 in the NAc $\left(F_{3,16}=196.50, P<0.0001\right)$, compared to GFP control (endogenous expression) in the absence of doxycycline and a 1.5-fold overexpression in presence of doxycycline, i.e., upon suppression of ectopic NZF-2b/7ZFMyt1 expression in the NAc $\left(F_{1,16}=104.48, P<0.0001\right)$. The ratios of control genes, e.g., cyclophilin or $\beta$-actin mRNA levels, remained unchanged over treated, untreated and control animals (data not shown). When injected with Lenti-7ZFMyt1-siRNAs in the NAc animals displayed a 5.7-fold decrease in NZF-2b/7ZFMyt1 mRNA in the NAc, compared to GFP controls under all regimens $\left(F_{3,16}=196.50, P<0.0001\right)$. This was expected as doxycycline blocks ectopic, lentivirus-mediated NZF-2b/7ZFMYT1 overexpression but not endogenously expressed NZF-2b/7ZFMYT1, while the NZF-2b/7ZFMYT1 silencers block both ectopic and endogenous NZF-2b/7ZFMYT1 expression. Finally animals co-injected Lenti-7ZFMyt1 and a mix of three Lenti-7ZFMyt1siRNAs in the NAc displayed expression similar to GFP controls in absence of doxycycline $\left(F_{3,16}=196.50, P<0.0001\right)$ and a 4.2 -fold decrease over GFP controls under doxycycline regimen $\left(F_{1,16}=104.48, \quad P<0.0001\right)$. Observation of some expression in Lenti-7ZFMyt1 treated animals under doxycycline regimen is clearly attributed to the slight leakiness of the Tet-off system used in our constructs.

Lentiviral injections into the NAc region lead to infection of the dopaminergic neurons projecting from the VTA. Retrograde Transport mediated by lentivirus has been amply documented, although it is far less efficient than, e.g. rabies or EEAV (Kitagawa et al., 2007; Mukherjee et al., 2007; Osten et al., 2006). Active lentiviral particles may therefore also be retro-transported to the VTA possibly leading to the expression of NZF-2b/7ZFMyt1, as observed in previous studies (Bahi et al., 2008; Boyer and Dreyer, 2007). To check whether this was the case, expression levels of NZF-2b/7ZFMyt1 in the VTA were quantified as mentioned for the NAc region. The results demonstrate that NZF-2b/7ZFMyt1 indeed is expressed, 

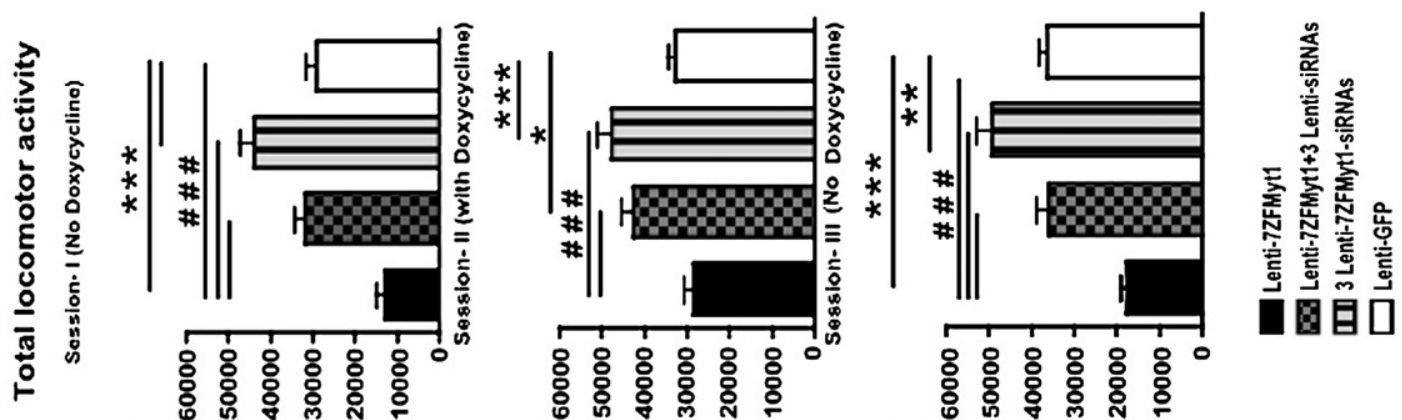

m

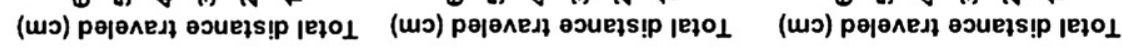
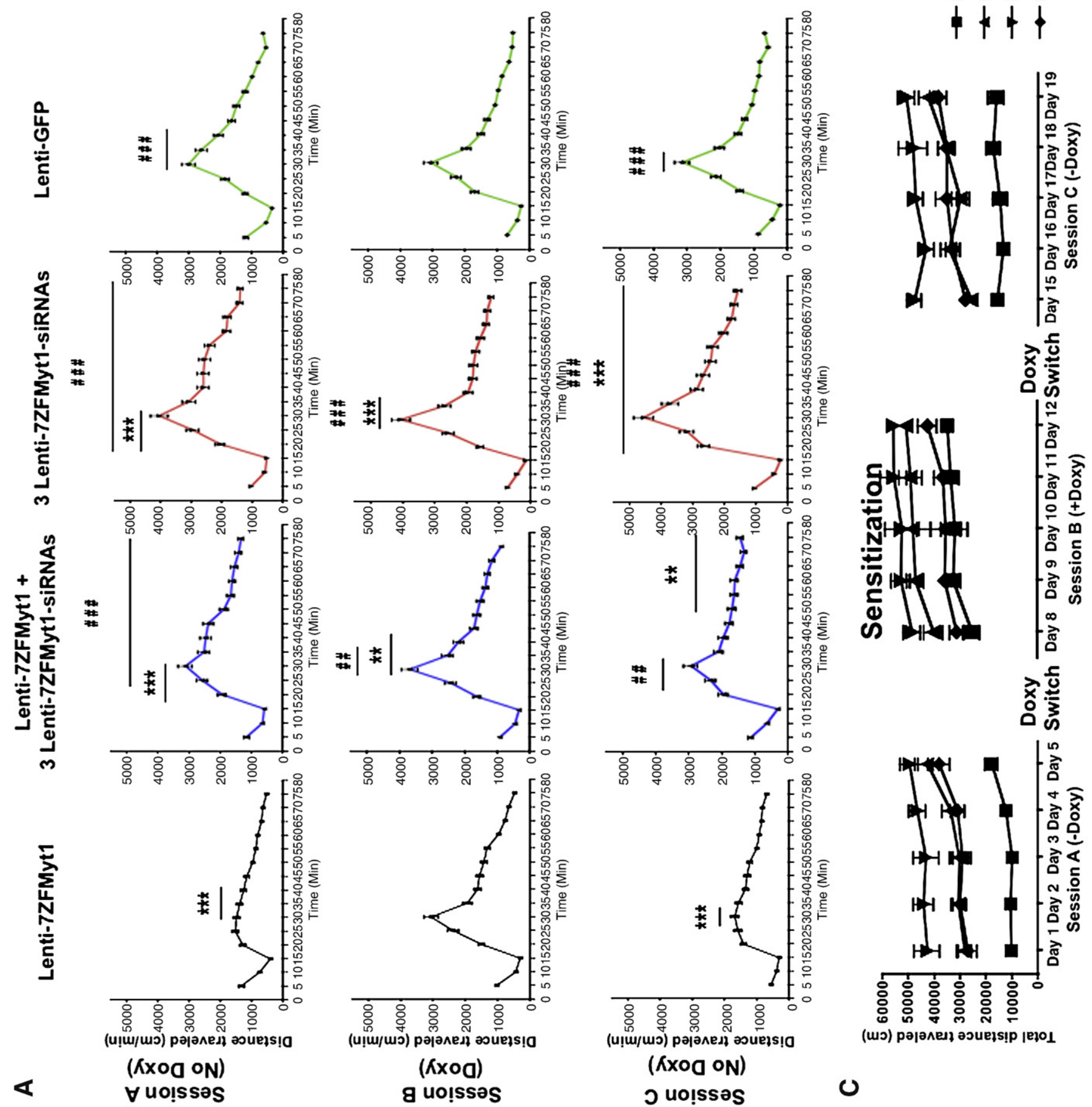

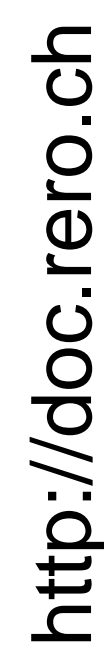


爻兘

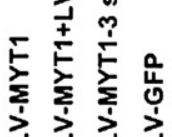

中 $中+$

0 
A NZF-2b/7ZFMyt1 expression profile in NAc after lentiviral injection in NAc-

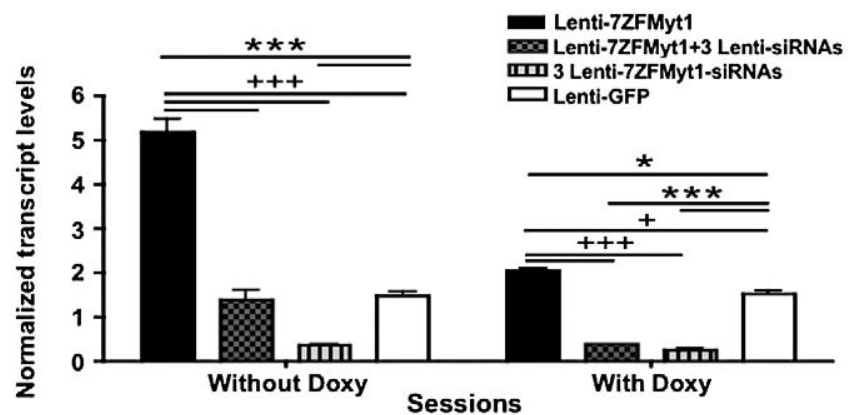

B NZF-2b/7ZFMyt1 expression profile in VTA after lentiviral injection in NAc

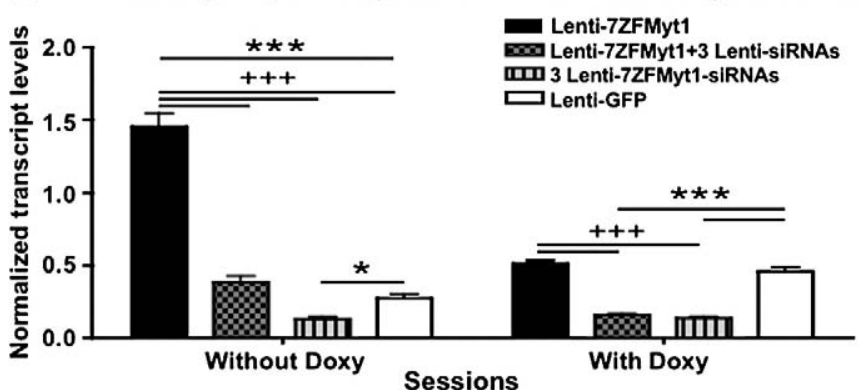

Fig. 3. In vivo expression of NZF-2b/7ZFMyt1 mRNA after injection of lenti-7ZFMyt1 and/or lenti-7ZFMyt1-siRNAs in the NAc. (A) NZF-2b/7ZFMyt1 expression in the NAc; (B) NZF-2b/7ZFMyt1 expression in the VTA. Quantification by qRT-PCR of NZF-2b/ 7ZFMYT1 mRNA transcript levels, either in the NAc (A) or in the VTA (B), from rats $(n=9)$ bilaterally injected into the NAc. Animals were stereotaxically injected with either lenti-7ZFMyt1 alone; lenti-7ZFMyt1 in combination with a mix of the three lenti7ZFMyt1 siRNAs; a mix of the three lenti-7ZFMyt1 siRNAs alone; or lenti-GFP (see Methods for details). Samples were retrieved after the end of the behavioral analysis (locomotor activity), either from animals fed without doxycycline or from animals fed doxycycline. Animals were sacrificed by decapitation $24 \mathrm{~h}$ after the last injection, the NAc region was micro-dissected and used for total RNA isolation, CDNA preparation and qRT-PCR. Expression levels were calculated relative to cyclophilin-D or $\beta$-actin. ${ }^{*} P<0.05 ; * * P<0.01 ; * * * P<0.001$ represent values significantly different from lentiGFP injected rats, and ${ }^{+} P<0.05 ;{ }^{++} P<0.01 ;{ }^{++} P<0.001$ represent values significantly different from lenti-7ZFMyt1 injected rats, by two-way ANOVA, Bonferroni post hoc tests.

albeit to a lesser level compared to NAc region as shown in Fig. 3B. In the absence of doxycycline, animals injected with Lenti-7ZFMyt1 in the NAc displayed a 5.1-fold increase in NZF-2b/7ZFMyt1 expression (compared to GFP controls) in the VTA $\left(F_{3,16}=188.48\right.$, $P<0.0001)$, but with doxycycline treatment this difference is abolished $\left(F_{1,16}=80.52, P<0.0001\right)$. In contrast animals injected Lenti-7ZFMyt1-siRNAs in the NAc displayed a 2-fold down-regulation of NZF-2b/7ZFMyt1 expression in the VTA, which was not modified by doxycycline regimen. The results from immunohistochemistry studies (below) clearly confirm that the expression seen in VTA can be attributed to retrograde transport and not to diffusion of viral particles.
NZF-2b/7ZFMyt1 localizes into the nucleus of the neurons in the NAc region after lentiviral mediated over expression

The changes in NZF-2b/7ZFMyt1 mRNA expression were further correlated at the protein level by means of immunohistochemistry (Fig. 4). Animals treated with Lenti-7ZFMyt1 displayed high level of expression of NZF-2b/7ZFMyt1 in the NAc. Under the same conditions, doxycycline treatment induced a strong suppression $(>90 \%)$ of ectopic NZF-2b/7ZFMyt1 expression in the NAc (Fig. 4A). This showed a doxycycline-mediated changes in NZF-2b/7ZFMyt1 expression in 7ZFMyt1-treated animals. GFP-treated animals or animals injected with Lenti-7ZFMyt1-siRNAs displayed no visible NZF-2b/7ZFMyt1 expression under all regimens, as the anti-Myc-antibody recognizes only the Myc-tagged, ectopically expressed NZF-2b/7ZFMyt1 protein and not the endogenous form (Fig. 4A). Since there was little difference observed between the expression levels of NZF-2b/ 7ZFMyt1 mRNA level between different doxycycline regimens in the control GFP group, it is reasonable to believe that the endogenous protein levels does not change between the groups, under the same chronic cocaine treatment. The animals injected with Lenti-7ZFMyt1 together with Lenti-7ZFMyt1-siRNAs displayed lesser NZF-2b/ 7ZFMyt1 expression that was fully suppressed under doxycycline regimen (Fig. 4A).

As NZF-2b/7ZFMyt1 - a known suppressive transcription factor displayed inhibitory effects on cocaine sensitivity, we wanted to check whether this suppression is via its transcriptional function. Immunolocalization studies were performed to check whether ectopic NZF-2b/ 7ZFMyt1 (Myc-tagged) localizes to nucleus or cytoplasm of the neurons (Fig. 4B). Double immunostaining with the neuron-specific marker NeuN under high magnification of NZF-2b/7ZFMyt1-expressing neurons shows that 7ZFMyt1-Myc expression appears as speckles within the nucleus (Fig. 4B), suggestive of an association of NZF-2b/7ZFMyt1 with a function that is spatially segregated into discrete nuclear domains similar to the sites of active transcription, which corresponds to the data obtained from immunostudies by Nielsen et al., 2004. Similar results were observed with antibody against native NZF-2b/ 7ZFMyt1 protein (Fig. 4C), which will recognize both the endogenous and ectopically expressed NZF-2b/7ZFMyt1 protein. Double immunostaining of ectopic Myc-tagged 7ZFMyt1 (with anti-Myc antibodies) and GFAP (with anti-GFAP antibodies) shows that ectopic NZF-2b/7ZFMyt1 is absent in GFAP-positive cells and is poorly co-localized in astrocytes (Fig. 4D). Furthermore the nuclear localization was confirmed by double staining using non-specific nuclei staining Hoechst-33342 and anti-Myt1 antibody, which shows that NZF-2b/7ZFMyt1 expression is restricted to cell type-specific nuclei (Fig. 4E), in this case neuronal as confirmed with NeuN staining. Double staining for antiMyt1 and anti-Myc (Fig. 4F), confirmed the colocalization and specificity of the antibodies used. The restricted, lentiviral mediated expression of ectopic NZF-2b/7ZFMyt1 within the NAc is clearly visualized with DAB (brown) staining and low resolution light microscopy (Fig. 4G), using anti-Myc antibody which localizes within the NAc region, identified by the proximity to aca (Commissure anterior Anterior part).

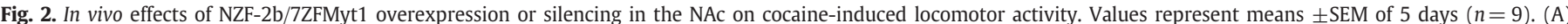

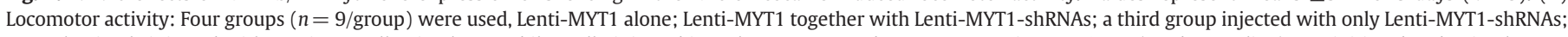

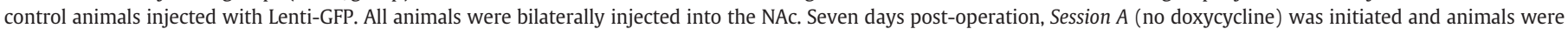

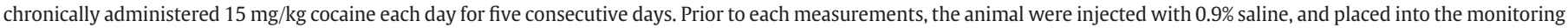

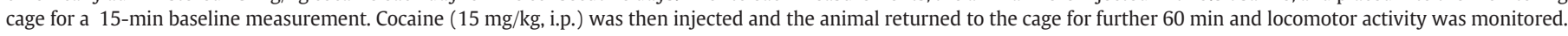

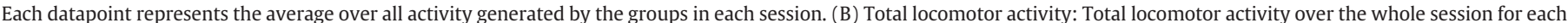

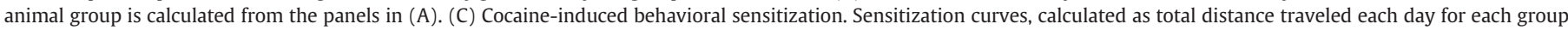

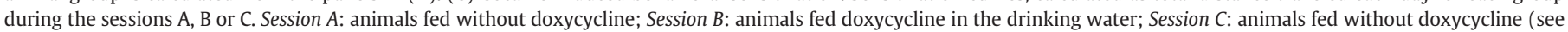

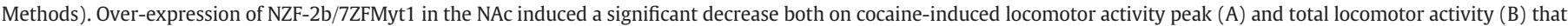

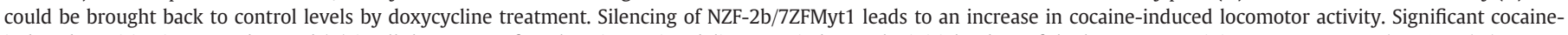

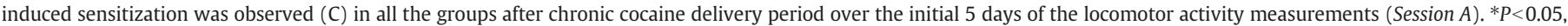

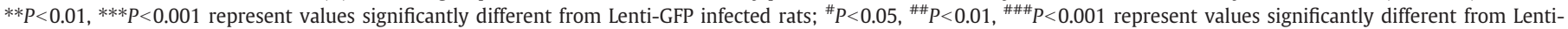
7ZFMyt1 injected rats; two-way ANOVA, Bonferroni post hoc tests. 


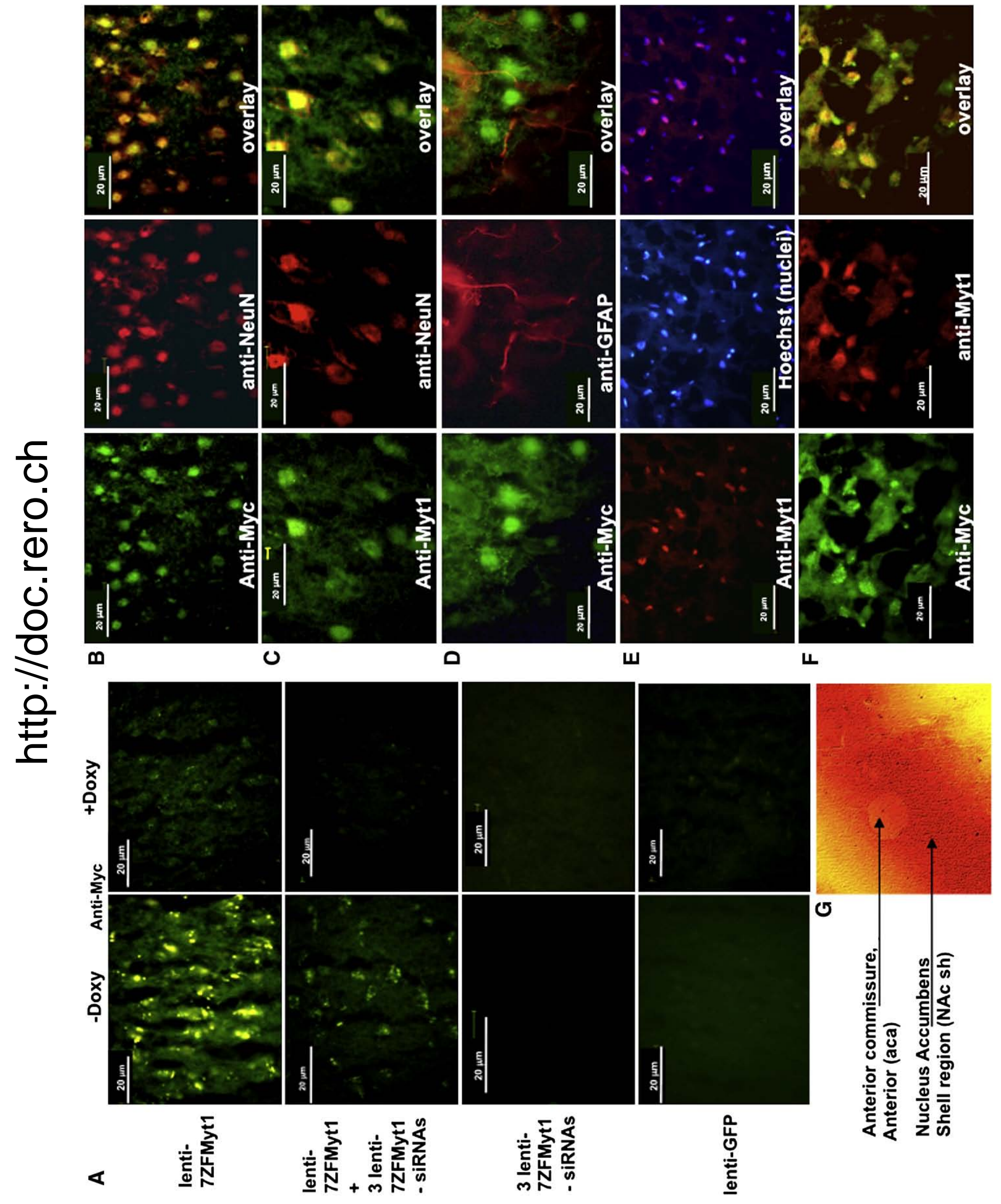


NZF-2b/7ZFMyt1 overexpression in the NAc induces changes in the expression levels of REST1, NAC1 and BDNF

Changes in NZF-2b/7ZFMyt1 expression might result in cocainemediated changes in down-stream signaling. Analysing all the possible genes modulated upon NZF-2b/7ZFMyt1 overexpression is beyond the scope of this study. Here, we focused on the BDNF (brain derived neurotrophic factor), REST1 and the transcription factor NAC1, a well studied suppressor of cocaine sensitivity (Mackler et al., 2000). As a matter of fact, it has been shown that NZF-2b/7ZFMyt1 inhibits the expression of MeCP2 (Liu et al., 2009), which in turn may represses REST1 and CoREST expression (Abuhatzira et al., 2007). Furthermore expression of REST and CoREST may inhibit BDNF expression levels, as shown in Rett syndrome (Abuhatzira et al., 2007). Also NZF-2b/7ZFMyt1 itself has putative conserved functional binding site modules in BDNF. Together this suggested that NZF-2b/ 7ZFMyt1 overexpression might lead to induction of REST1 expression and probably inhibition of BDNF levels. To check the hypothesis, the expression of these effectors was further tested in relation to NZF-2b/ 7ZFMyt1 expression (Fig. 5).

We observed that REST1 expression is induced significantly (ca. 2.5-fold) upon chronic cocaine administration, compared to salinetreated animals (data not shown). Furthermore, we observed that REST1 displays significant expression changes that correlate with expression changes of NZF-2b/7ZFMyt1 (Fig. 5A). In the different lentivirus-treated animal groups, doxycycline regimen that modifies NZF-2b/7ZFMyt1 expression also affects REST1 expression (Fig. 5A; $\left.F_{3,16}=90.67, P<0.0001\right)$. In the absence of doxycycline a 1.8 -fold induction of REST1 was observed in Lenti-7ZFMyt1 treated animals, compared to GFP-treated animals, where REST1 is unaltered even in the presence of doxycycline (Fig. $5 A ; F_{3,16}=289.42, P<0.0001$ ). Local suppression of NZF-2b/7ZFMyt1 expression in Lenti-siRNAs treated animals results in basal REST1 expression level in the presence or absence of doxycycline, comparable to saline treated naive animals. Under doxycycline regimen the levels of REST1 expression dropped significantly in both the Lenti-7ZFMyt1 treated group and the group treated with Lenti-7ZFMyt1 together with the three Lenti-7ZFMyt1siRNA (Fig. 5A). No change was observed in the group treated with Lenti-7ZFMyt1-siRNAs alone and or in the Lenti-GFP group. Under these conditions, Lenti-7ZFMyt1 treated animals display 40\% reduction in REST1 expression compared to GFP-treated controls (Fig. 5A; $\left.F_{1,16}=250.03, P<0.0001\right)$. Doxycycline regimen did not modify the REST1 expression profile of the Lenti-7ZFMyt1-siRNA group (as expected, since expression of siRNAs was not regulatable).

NZF-2b/7ZFMyt1 expression in the NAc strongly induces NAC1 in that brain region, and doxycycline regimen that modifies NZF2b/7ZFMyt1 expression in lentivirus-treated animals also affects NAC1 expression (Fig. 5B $F_{3,16}=37.91, P<0.0001$ ). In the absence of doxycycline, 7ZFMyt1-overexpressing animals display a 2.4-fold increase in NAC1 expression compared to GFP-treated controls (Fig. 5B; $\left.F_{3,16}=55.47, P<0.0001\right)$. Doxycycline did not affect NAC1 expression profile in lenti-GFP-treated animals, but in Lenti-7ZFMyt1 treated animals it induced a suppression of NAC1, by suppressing local ectopic expression of NZF-2b/7ZFMyt1. In contrast, suppression of NZF-2b/ 7ZFMyt1 in animals treated with Lenti-7ZFMyt1-siRNAs results in 2-fold decreased expression of NAC1 compared to Lenti-GFP group under the same cocaine treatment conditions, and was not significantly modified under doxycycline regimen (Fig. 5B; $F_{1,16}=118.51$, $P<0.0001$ ).

Furthermore NZF-2b/7ZFMyt1 induction in cocaine-treated animals leads to potent inhibition of BDNF. In Lenti-7ZFMyt1 treated animals we observed a strong inhibition of BDNF mRNA levels (Fig. 5C; $F_{3,24}=35.34, P<0.0001$ ), the lenti-7ZFMyt1 treated group yielding a 3.8 fold decreased expression in the absence of doxycycline $\left(F_{3,24}=200.93, P<0.0001\right)$, compared to lenti-GFP group (Fig. 5C). As observed in NAC1 and REST1, doxycycline treatment did not affect the expression level of BDNF in lenti-GPF and Lenti-7ZFMyt1-siRNAs only groups; but in the Lenti-7ZFMyt1 and Lenti-7ZFMyt1+Lenti7ZFMyt1-siRNAs groups an increase in BDNF expression was observed (Fig. 5C; $F_{1,24}=78.15, P<0.0001$ ). Interestingly suppression of NZF-2b/7ZFMyt1 (in animals treated with Lenti-7ZFMyt1-siRNAs alone) results in 1.8-fold increased expression of BDNF compared to Lenti-GFP group under the same cocaine treatment conditions.

\section{Discussion}

Chronic abuse of cocaine causes neuroadaptive changes in the Nucleus Accumbens (NAc) and the Ventral Tegmental Area (VTA) (Nestler, 2000). Our data show that cocaine induces elevated expression of NZF-2b/7ZFMyt1 (Neural-Zinc-Finger transcription factor 2b/7-Zinc Finger Myt1), particularly in the mesolimbic dopaminergic pathway, resulting in an 11-fold increase in the nucleus accumbens and a 30 -fold increase in the hippocampus. This is suggestive of a role for NZF2b/7ZFMyt1 in drug-associated behavior and reward, and also maybe in learning and memory and cue-related behavior. However a clarification of that role needs further investigations. NZF-2b/7ZFMyt1 is normally found in neural precursors and in some subpopulations of mature neurons (Bellefroid et al. 1996; Kim et al. 1997), and it is also expressed in developing oligodendrocytes (Armstrong et al. 1995). NZF-2b/7ZFMyt1 interacts with three isoforms of Sin3B, which mediates transcriptional repression by binding to histone deacetylases HDAC1 and HDAC2 (Romm et al., 2005).

Furthermore, we found that localized expression changes of NZF-2b/7ZFMyt1 in the NAc significantly affect cocaine-mediated behavior. Chronic cocaine administration can produce tolerance or sensitization to locomotor activating effects, depending on the

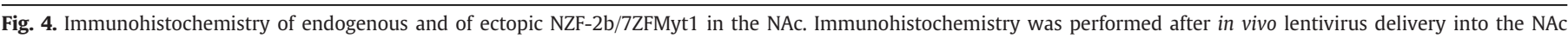

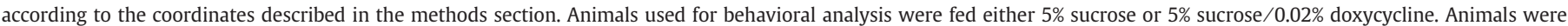

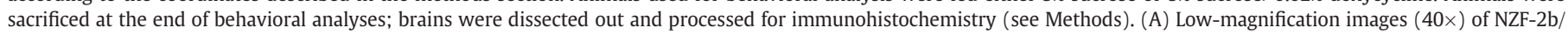

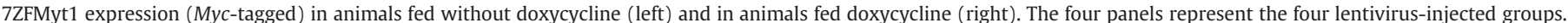

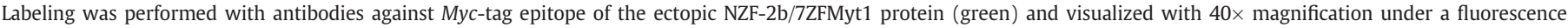

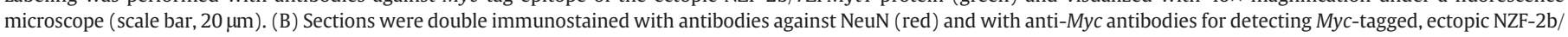

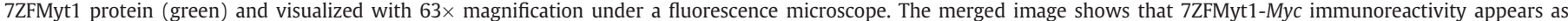

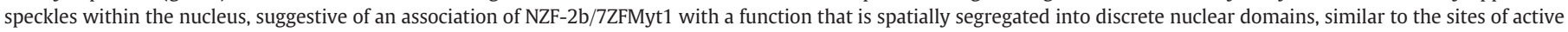

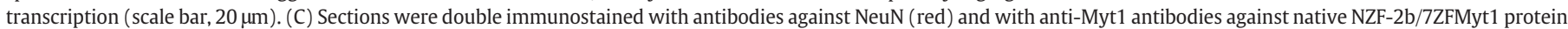

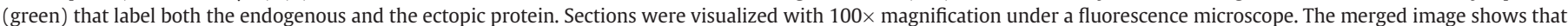

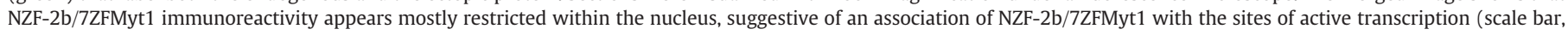

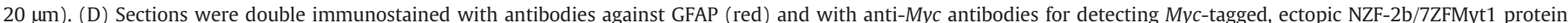

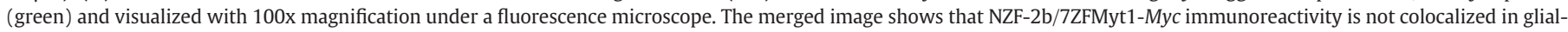

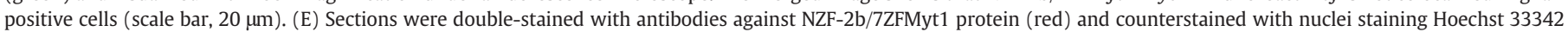

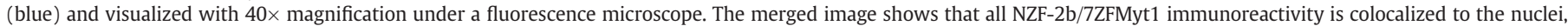

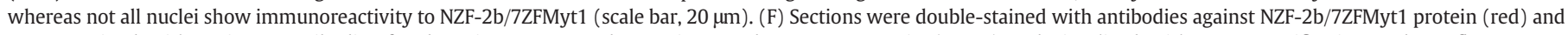

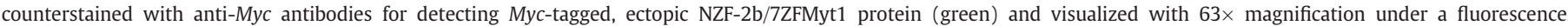

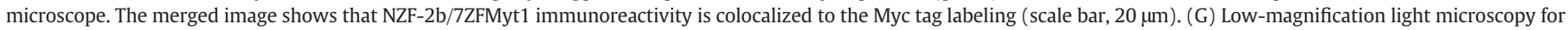
expression of NZF-2b/7ZFMyt1-Myc in the NAc. Sections were stained with anti-Myc antibodies and revealed by HRP-DAB (brown). Pictures were taken at $2.5 \times$ magnification.
} 
A REST1 expression profile in NAc after lenti-7ZFMyt1 injection

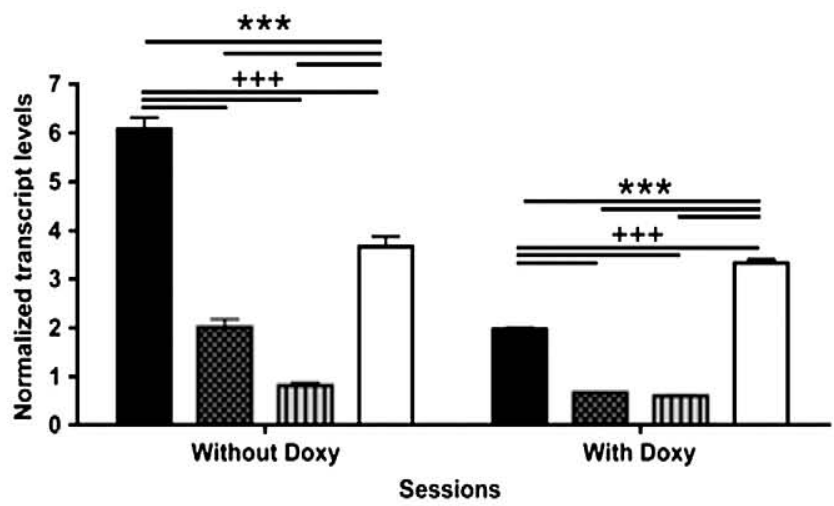

B NAC1 expression profile in NAc after lenti-7ZFMyt1 injection

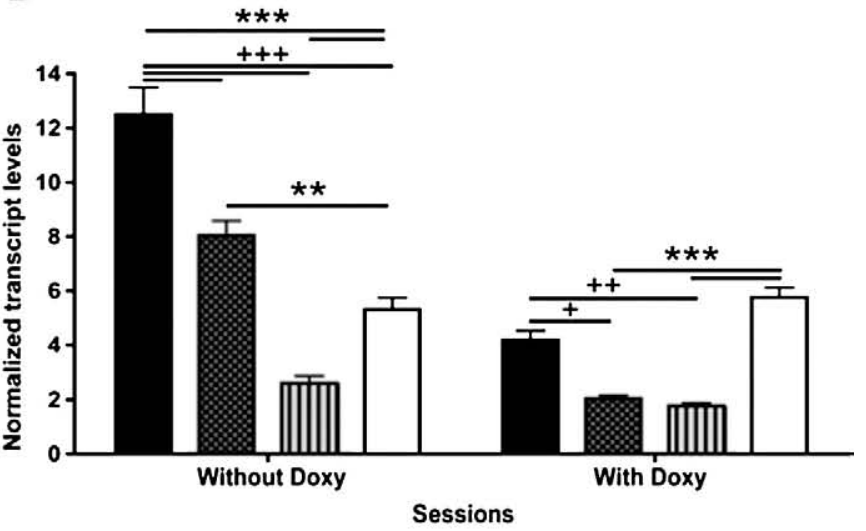

C BDNF expression profile in NAc after lenti-7ZFMyt1 injection
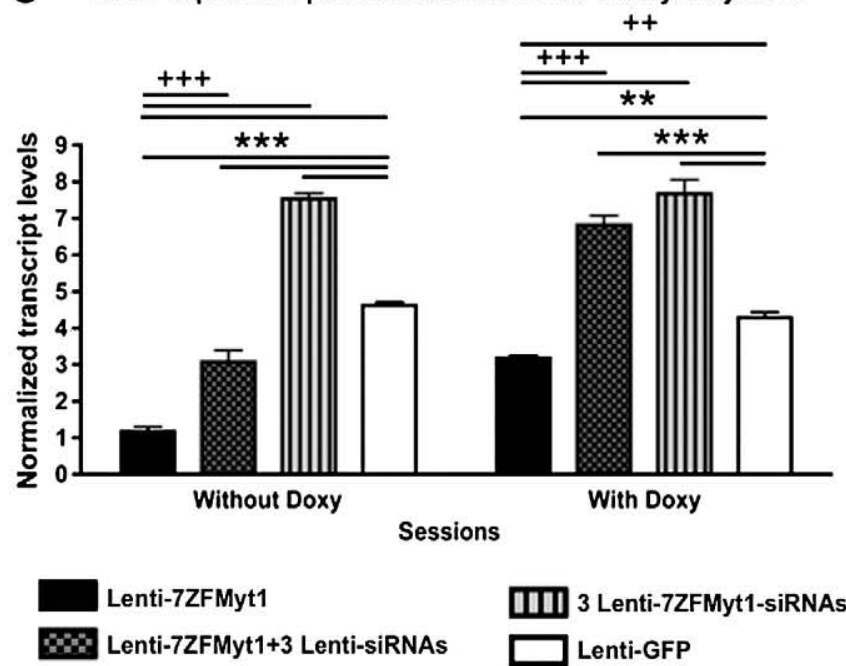

IIII 3 Lenti-7ZFMyt1-siRNAs Lenti-GFP

Fig. 5. Effect of NZF-2b/7ZFMyt1 expression and silencing in the Nucleus Accumbens (NAc) on the mRNA levels of REST1, NAC1 and BDNF. Quantification of REST1 (A), NAC1 (B) and BDNF (C) transcript levels by qRT-PCR from rats $(n=9)$ bilaterally injected into the NAc with either lenti-7ZFMyt1 alone, lenti-7ZFMyt1 in combination with a mix of three lenti-7ZFMyt1 siRNAs, a mix of three lenti-7ZFMyt1 siRNAs alone, or lenti-GFP (see Methods). Samples were retrieved at the end of behavioral analysis (locomotor activity) from animals fed either in the presence or in absence of doxycycline. $24 \mathrm{~h}$ after the last injection, animals were sacrificed by decapitation and the NAc was microdissected and used for total RNA isolation, cDNA preparation and qRT-PCR. Expression levels were calculated relative to cyclophilin-D or $\beta$-actin. Overexpression of NZF-2b/ 7ZFMyt1 led to an increase in expression levels of REST1 (A) and NAC1 (B) and to a decrease in expression levels of BDNF (C). Doxycycline regimen that inhibits ectopic NZF-2b/7ZFMyt1 expression results in a significant decrease in REST1 (A) and NAC1 levels (B), but increases BDNF levels (C). $* P<0.05$; $* * P<0.01 ; * * * P<0.001$ represent values significantly different from lenti-GFP injected rats, and ${ }^{+} P<0.05$; ${ }^{++} P<0.01 ;{ }^{+++} P<0.001$ represent values significantly different from lenti-7ZFMyt 1 injected rats, by two-way ANOVA, Bonferroni post hoc tests. treatment paradigm and can be influenced by gene expression changes in the reward circuitry (Nestler, 2000). Selective induction of NZF-2b/7ZFMyt1 expression in the NAc significantly decreases an animal's responsiveness to the locomotor-activating effects of cocaine, whereas local silencing of NZF-2b/7ZFMyt1 results in hyperlocomotion. Similar observations have been described with overexpression of other genes, e.g., the D3R DA receptor (Bahi et al., 2005), and may be due to local compensatory mechanisms following drug injections (Kalivas and Nakamura, 1999). These results indicate that NZF-2b/7ZFMyt1 may mediate some of the persistent neural and behavioral plasticity that accompanies chronic drug exposure.

Continuous cocaine administration produces a rapid sensitization that is lost over the course of the treatment period (Nestler, 2000). Behavioral sensitization arise from neuroadapations in multiple brain nuclei (Vanderschuren and Kalivas, 2000) Our results showing changes in NZF-2b/7ZFMyt1 expression resulting in apparent cocaine-induced sensitization in the first session (session A) but not in subsequent sessions indicate the differential involvement of the accumbens vs. other brain regions in the induction or expression of cocaine sensitization. Moreover the lesser degree of sensitization observed after NZF-2b/7ZFMyt1 knock-down indicates a ceiling effect as the initial cocaine-induced locomotion was already strong enough that further increase was probably not possible. Tolerance and sensitization to the locomotor-activating effects of cocaine can exist simultaneously, since they are mediated by separate mechanisms (Izenwasser and French, 2002). Our results show that NZF-2b/ 7ZFMyt1 might be playing an important role in governing the animal's responsiveness in these disparate mechanisms.

Interestingly we observed that NZF-2b/7ZFMyt1 overexpression mediates increased expression of the transcription factors NAC1 and REST1 (RE1-silencing transcription factor-1) in the NAc, which can account for the decreased sensitivity to cocaine's rewarding effects. NAC1 is a cocaine-regulated POZ/BTB protein that is induced by cocaine selectively in the NAc and that interacts with histone deacetylases HDAC3 and HDAC4 (Korutla et al., 2005, 2007). The other factor affected by NZF-2b/7ZFMyt1, REST1, is a transcriptional repressor that represses neuronal gene transcription and is required for the acquisition of the differentiated functional neuronal phenotype during early development (Armisen et al., 2002). REST1 targets genes that are expressed in neurons (Hohl and Thiel 2005) and silences transcription of numerous neuron-specific genes in nonneuronal cells via recruitment of co-repressor complexes, Co-REST, Sin3A, HDAC1 and HDAC2, thus strongly affecting chromatin remodeling (Belyaev et al., 2004), to maintain a repressive chromatin environment in neural stem cells (Greenway et al., 2006). Induction of REST1 following NZF-2b/7ZFMyt1 expression after chronic cocaine may be an important factor for behavioral and cognitive alterations observed upon drug administration. Together NZF-2b/7ZFMyt1triggered expression of NAC1 and REST1 might provide mechanistic insight into how drugs of abuse can direct chromatin-modifying enzymes to specific genes. Indeed cocaine regulation of chromatinmodifying enzymes is currently considered to play a central function in shaping the addicted state (Tsankova et al., 2007). Complex epigenetic mechanisms which regulate gene activity have long-lasting effects within mature neurons.

Our findings that NZF-2b/7ZFMyt1 induces the expression of REST is supported by earlier studies showing that NZF-2b/7ZFMyt1 inhibits the expression of MeCP2 (Liu and Francke, 2006), which in turn may repress REST and CoREST expression (Abuhatzira et al., 2007). MeCP2 is a multifunctional protein involved in the modulation of chromatin structure and in alternate splicing mechanisms. NZF-2b/7ZFMyt1-binding sites are present in the MECP2 promoter, specifically between -553 and -681 as well as in enhancers F11 and F21 and silencer F13 (Liu and Francke, 2006), and thus NZF-2b/ 7ZFMyt1 may be part of a complex that keeps MECP2 expression low. Furthermore elevated levels of REST and CoREST (in the brain of Rett 
syndrome patients and in MeCP2 deficient mice) results in downregulation of BDNF, apparently by their binding to the RE1 element located between the first two promoters of the BDNF gene. Several studies described the crucial function of BDNF and TrkB in cocaine reward and relapse (Kalivas and O'Brien, 2008; Nestler 2000; Thomas et al. 2008; Bahi et al., 2008). Interestingly, the NTRK2 gene that encodes TrkB, the BDNF receptor, was overexpressed in MeCP2 deficient human and mouse brains either directly or as an attempt to compensate for BDNF deficiency (Abuhatzira et al., 2007). Our present finding implicate that NZF-2b/7ZFMyt1 plays an important role in induction of REST leading to downregulation of BDNF. Indeed we observe that NZF-2b/7ZFMyt1 overexpression leads to BDNF suppression. In addition, NZF-2b/7ZFMyt1 interacts with Lingo1 (Llorens et al., 2008), a component of the Nogo receptor complex that mediates intracellular signaling in response to myelinassociated inhibitors (MAIs). This interaction is responsible for the intracellular localization and regulation of transcriptional activity of NZF-2b/7ZFMyt1, whereby Lingo1 causes cytoplasmic localization of NZF-2b/7ZFMyt1 (Llorens et al., 2008) and transcriptional inactivation. Interestingly BDNF induces expression of Lingo 1 (Trifunovski et al., 2004). We propose a model wherein chronic cocaine exposure results in gradual accumulation of NZF-2b/7ZFMyt1 in the NAc (Fig. 6), where a dynamic regulation between NZF-2b/7ZFMyt1 and BDNF might determine key events in the reward pathway. Thus

A

\section{Chronic cocaine}

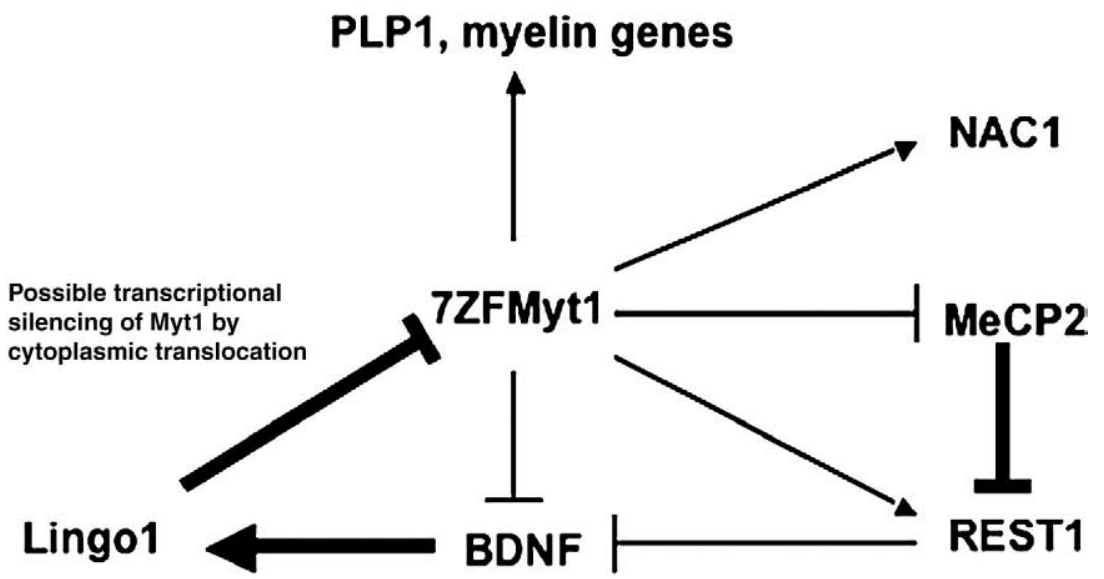

B

Lentiviral mediated NZF-2b/7ZFMyt1 ectopic overexpession

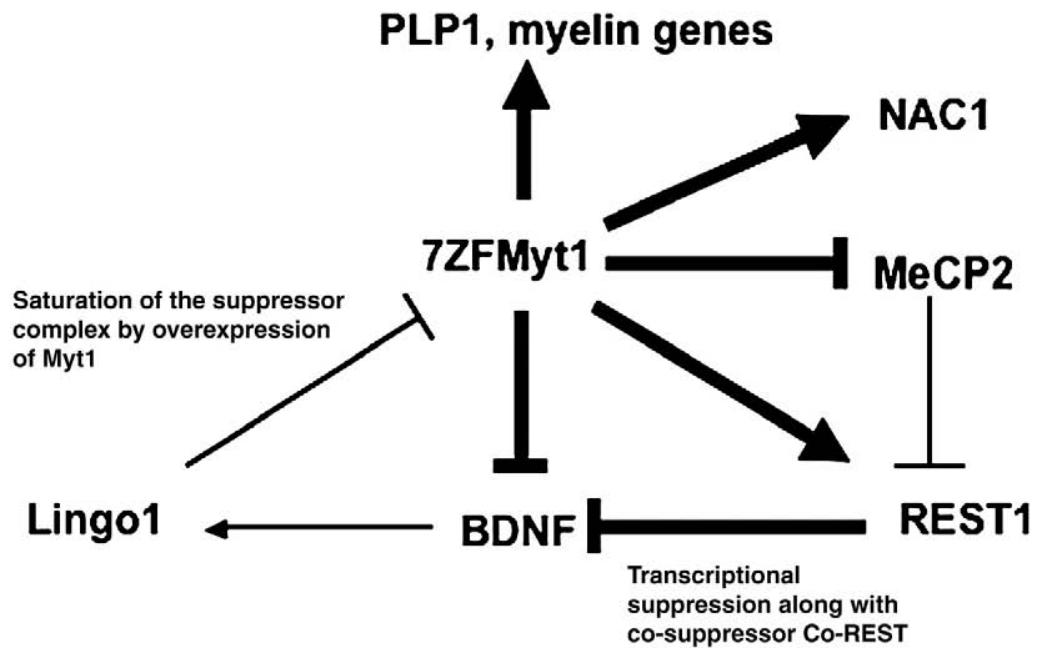

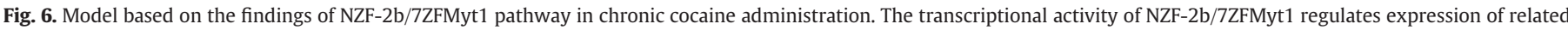

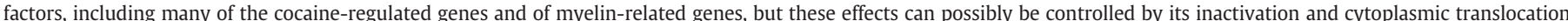

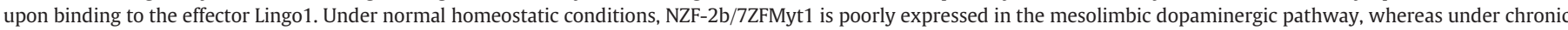

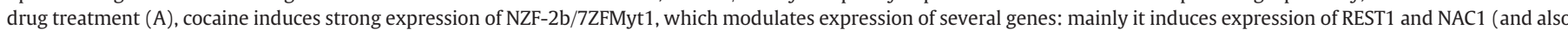

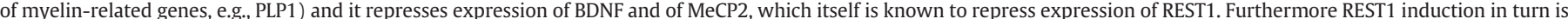

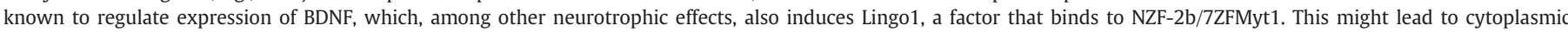

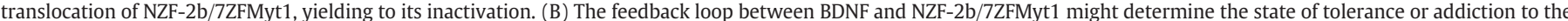

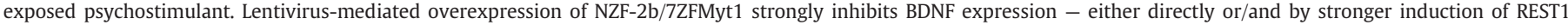
expression (see text for details). 
NZF-2b/7ZFMyt1 expression might be involved in titrating behavioral responses to psychostimulants through a complex series of downstream transcriptional events, involving the induction (NAC1 \& REST1 expression) or repression (BDNF expression) of several target genes. Future studies aimed at identifying the factors that determine NZF-2b/7ZFMyt1 effects on target genes, might shed light on this complex pathway. Clearly NAC1 and REST1 may be just two of the many targets regulated by NZF-2b/7ZFMyt1 in the NAc. Based on these observations, our study sheds light on the little known pathway in this brain region, which might lead to addiction-like behavior.

In conclusion, we show that NZF-2b/7ZFMyt 1 may be an important factor involved in phenotypic changes induced by chronic cocaine administration. So far it has been difficult to identify the molecular mechanisms that underlie such stable changes in gene expression. Our observations on the functional implication of NZF-2b/7ZFMyt1 provides an alternate insight into the basic transcriptional and epigenetic mechanisms involved in the desensitization of genes in drug administration, which may facilitate the development of more successful drug treatment strategies.

\section{Author disclosures}

This study was supported by Swiss National Foundation grants 3100-059350 and 3100AO-100686 (JLD). The SNF had no further role in study design; in the collection, analysis and interpretation of data; in the writing of the report; and in the decision to submit the paper for publication.

JLD designed the study. VC performed the analyses and managed the literature searches. VC and JLD undertook the data analysis. VC wrote the first draft of the manuscript. JLD wrote the final version of the manuscript. All authors contributed to and have approved the final manuscript.

All authors declare that they have no conflicts of interest.

\section{Acknowledgments}

The authors are grateful to Dr F. Boyer and Dr A. Bahi for critical comments and to Mrs C. Deforel-Poncet for skillful assistance.

\section{References}

Abuhatzira, L., Makedonski, K., Kaufman, Y., Razin, A., Shemer, R., 2007. MeCP2 deficiency in the brain decreases BDNF levels by REST/CoREST-mediated repression and increases TRKB production. Epigenetics 2 (4), 214-222.

Armisen, R., Fuentes, R., Olguín, P., Cabrejos, M.E., Kukuljan, M., 2002. Repressor element-1 silencing transcription/neuron-restrictive silencer factor is required for neural sodium channel expression during development of Xenopus. J. Neurosci. 22 (19), 8347-8351.

Armstrong, R.C., Kim, J.G., Hudson, L.D., 1995. Expression of myelin transcription factor I (MyTI), a "zinc-finger" DNA-binding protein, in developing oligodendrocytes. Glia 14 (4), 303-321.

Bahi, A., Dreyer, J.L., 2005. Cocaine-induced expression changes of axon guidance molecules in the adult rat brain. Mol. Cell. Neurosci. 28, 275-291.

Bahi, A., Boyer, F., Kafri, T., Dreyer, J.L., 2004. CD81-induced behavioural changes during chronic cocaine administration. In vivo gene delivery with regulatable Lentivirus. Eur. J. Neurosci. 19, 1621-1633.

Bahi, A., Boyer, F., Kolira, M., Dreyer, J.L., 2005. In vivo gene silencing of CD81 by lentiviral expression of small interference RNAs suppresses cocaine-induced behaviour. J. Neurochem. 92, 1243-1255.

Bahi, A., Boyer, F., Dreyer, J.L., 2008. Role of accumbens BDNF and TrkB in cocaineinduced psychomotor sensitization, conditioned-place preference, and reinstatement in rats. Psychopharmacology 199 (2), 169-182.

Bellefroid, E.J., Bourguignon, C., Hollemann, T., Ma, Q., Anderson, D.J., Kintner, C., Pieler, T.X., 1996. MyT1, a Xenopus C2HC-type zinc finger protein with a regulatory function in neuronal differentiation. Cell 87, 1191-1202.
Belyaev, N.D., Wood, I.C., Bruce, A.W., Street, M., Trinh, J.B., Buckley, N.J., 2004. Distinct RE-1 silencing transcription factor-containing complexes interact with different target genes. J. Biol. Chem. 279 (1), 556-561.

Boyer, F., Dreyer, J.L., 2007. Alpha-synuclein in the nucleus accumbens induces changes in cocaine behaviour in rats. Eur. J. Neurosci. 26, 2764-2776.

Cha, X.Y., Pierce, R.C., Kalivas, P.W., Mackler, S.A., 1997. NAC-1, a rat brain mRNA, is increased in the nucleus accumbens three weeks after chronic cocaine selfadministration. J. Neurosci. 17 (18), 6864-6871.

Conrad, K.L., Tseng, K.Y., Uejima, J.L., Reimers, R.M., Heng, L.J., Shaham, Y., Marinelli, M., Wolf, M.E., 2008. Formation of accumbens GluR2-lacking AMPA receptors mediates incubation of cocaine craving. Nature 454, 118-121.

Deloukas, P., Matthews, L.H., Ashurst, J., Burton, J., Gilbert, J.G.R., Jones, M., Stavrides, G., Almeida, J.P., Babbage, A.K., Bagguley, C.L., Bailey, J., Barlow, K.F., Bates, K.N., Beard, L.M., Beare, D.M., Beasley, O.P., Bird, C.P., Blakey, S.E., Bridgeman, A.M., Brown, A.J., Buck, D., Burrill, W.D., Butler, A.P., Carder, C., Carter, N.P., Chapman, J.C., Clamp, M., Clark, G., Clark, L.N., Clark, S.Y., Clee, C.M., Clegg, S., Cobley, V.E., Collier, R.E., Connor, R.E., Corby, N.R., Coulson, A., Coville, G.J., Deadman, R., Dhami, P.D., Dunn, M., Ellington, A.G., Frankland, J.A., Fraser, A., French, L., Garner, P., Grafham, D.V., Griffiths, C., Griffiths, M.N.D., Gwilliam, R., Hall, R.E., Hammond, S., Harley, J.L., Heath, P.D., Ho, S., Holden, J.L., Howden, P.J., Huckle, E., Hunt, A.R., Hunt, S.E., Jekosch, K., Johnson, C.M., Johnson, D., Kay, M.P., Kimberley, A.M., King, A., Knights, A., Laird, G.K., Lawlor, S., Lehvaeslaiho, M.H., Leversha, M.A., Lloyd, C., Lloyd, D.M., Lovell, J.D., Marsh, V.L., Martin, S.L., McConnachie, L.J., McLay, K., McMurray, A.A., Milne, S.A., Mistry, D., Moore, M.J.F., Mullikin, J.C., Nickerson, T., Oliver, K., Parker, A. Patel, R., Pearce, T.A.V., Peck, A.I., Phillimore, B.J.C.T., Prathalingam, S.R., Plumb, R.W., Ramsay, H., Rice, C.M., Ross, M.T., Scott, C.E., Sehra, H.K., Shownkeen, R., Sims, S., Skuce, C.D., Smith, M.L., Soderlund, C., Steward, C.A., Sulston, J.E., Swann, R.M., Sycamore, N., Taylor, R., Tee, L., Thomas, D.W., Thorpe, A., Tracey, A., Tromans, A.C., Vaudin, M., Wall, M., Wallis, J.M., Whitehead, S.L., Whittaker, P., Willey, D.L., Williams, L., Williams, S.A., Wilming, L., Wray, P.W., Hubbard, T., Durbin, R.M., Bentley, D.R., Beck, S., Rogers, J., 2002. The DNA sequence and comparative analysis of human chromosome 20. Nature 414 (6866), 865-871.

Greenway, D.J., Streeta, M., Jeffries, A., Buckley, N.J., 2006. RE1 silencing transcription factor maintains a repressive chromatin environment in embryonic hippocampal neural stem cells. Stem Cells 25 (2), 354-363.

Hohl, M., Thiel, G., 2005. Cell type-specific regulation of RE-1 silencing transcription factor (REST) target genes. Eur. J. Neurosci. 22 (9), 2216-2230.

Izenwasser, S., French, D., 2002. Tolerance and sensitization to the locomotor-activating effects of cocaine are mediated via independent mechanisms. Pharmacol. Biochem. Behav. 73 (4), 877-882.

Kalivas, P.W., Nakamura, M., 1999. Neural systems for behavioral activation and reward. Curr. Opin. Neurobiol. 9 (2), 223-227.

Kalivas, P.W., O'Brien, C., 2008. Drug addiction as a pathology of staged neuroplasticity. Neuropsychopharmacology 33 (1), 166-180.

Kalivas, P.W., Duffy, P., Mackler, S.A., 1999. Interrupted expression of NAC-1 augments the behavioral responses to cocaine. Synapse 33, 153-159.

Kikuno, R., Nagase, T., Ishikawa, K., Hirosawa, M., Miyajima, N., Tanaka, A., Kotani, H., Nomura, N., Ohara, O., 1999. Prediction of the coding sequences of unidentified human genes. XIV. The complete sequences of 100 new cDNA clones from brain which code for large proteins in vitro. DNA Res. 6 (3), 197-205.

Kim, J.G., Hudson, L.D., 1992. Novel member of the zinc finger superfamily: a C2-HC finger that recognizes a glia-specific gene. Mol. Cell. Biol. 12 (12), 5632-5639.

Kim, J.G., Armstrong, R.C., v Agoston, D., Robinsky, A., Wiese, C., Nagle, J., Hudson, L.D., 1997. Myelin transcription factor 1 (Myt1) of the oligodendrocyte lineage, along with a closely related $\mathrm{CCHC}$ zinc finger, is expressed in developing neurons in the mammalian central nervous system. J. Neurosci. Res. 50 (2), 272-290.

Kitagawa, R., Miyachi, S., Hanawa, H., Takada, M., Shimada, T., 2007. Differential characteristics of HIV-based versus SIV-based lentiviral vector systems: gene delivery to neurons and axonal transport of expressed gene. Neurosci. Res. 57 (4), 550-558.

Korutla, L., Wang, P.J., Mackler, S.A., 2005. The POZ/BTB protein NAC1 interacts with two different histone deacetylases in neuronal-like cultures. J. Neurochemistry 94 (3), 786-793.

Korutla, L., Degnan, R., Wang, P., Mackler, S.A., 2007. NAC1, a cocaine-regulated POZ/ BTB protein interacts with CoREST. J. Neurochem. 101 (3), 611-618.

Liu, H., Hu, Q., D'ercole, A.J., Ye, P., 2009. Histone deacetylase 11 regulates oligodendrocyte-specific gene expression and cell development in OL-1 oligodendroglia cells. Glia. 57 (1), 1-12.

Liu, J., Francke, U., 2006. Identification of cis-regulatory elements for MECP2 expression. Hum. Mol. Genet. 115 (11), 1769-1782.

Llorens, F., Gil, V., Iraola, S., Carim-Todd, L., Martí, E., Estivill, X., Soriano, E., del Rio, J.A., Sumoy, L, 2008. Developmental analysis of Lingo-1/Lern1 protein expression in the mouse brain: interaction of its intracellular domain with Myt11. Dev. Neurobiol. 68 (4), 521-541.

Lüscher, C., Bellone, C., 2008. Cocaine-evoked synaptic plasticity: a key to addiction? Nat. Neurosci. 11 (7), 737-738.

Mackler, S.A., Korutla, L., Cha, X.Y., Koebbe, M.J., Fournier, K.M., Bowers, M.S., Kalivas, P.W., 2000. NAC-1 is a brain POZ/BTB protein that can prevent cocaine-induced sensitization in the rat. J. Neurosci. 20 (16), 6210-6217.

Matsushita, F., Kameyama, T., Marunouchi, T., 2002. NZF-2b is a novel predominant form of mouse NZF-2/MyT1, expressed in differentiated neurons especially at higher levels in newly generated ones. Mech. Dev. 118 (1-2), 209-213.

McClung, C.A., Ulery, P.G., Perrotti, L.I., Zachariou, V., Berton, O., Nestler, E.J., 2004. DeltaFosB: a molecular switch for long-term adaptation in the brain. Mol. Brain Res. 132, 146-154.

Michna, L., Brenz-Verca, M., Dreyer, J.L., Wagner, G.C., 2002. Methods to examine molecular changes and behavioral effects of drug administration. Brain Res. Brain Res. Protoc. 9 (3), 181-196. 
Mukherjee, S., Lee, H.L.R., Pacchia, A.L., Ron, Y., Dougherty, J.P., 2007. A HIV-2-based self-inactivating vector for enhanced gene transduction. J. Biotechnol. 127 (4), 745-757.

Nagase, T., Ishikawa, K., Suyama, M., Kikuno, R., Hirosawa, M., Miyajima, N., Tanaka, A., Kotani, H., Nomura, N., Ohara, O., 1998. Prediction of the coding sequences of unidentified human genes: XII. The complete sequences of 100 new cDNA clones from brain which code for large proteins in vitro. DNA Res. 5 (6), 355-364.

Nestler, E.J., 2000. Genes and addiction. Nat. Genet. 26, 277-281.

Nielsen, J.A., Berndt, J.A., Hudson, L.D., Armstrong, R.C., 2004. Myelin transcription factor 1 (Myt1) modulates the proliferation and differentiation of oligodendrocyte lineage cells. Mol. Cell. Neurosci. 25 (1), 111-123.

Osten, P., Dittgen, T., Licznerski, P., 2006. Lentivirus-based genetic manipulations in neurons in vivo. In: Kittler, Josef $\mathrm{T}$. Moss, Stephen J. (Eds.), Frontiers in Neuroscience. The Dynamic Synapse: Molecular Methods in Ionotropic Receptor Biology. CRC Press, Taylor \& Francis Group, Boca Raton, FL.

Paxinos, G., Watson, C., 1998. The Rat Brain in Stereotaxic Coordinates, Fourth Edition. Academic Press, New York.

Romm, E., Nielsen, J.A., Kim, J.G., Hudson, L.D., 2005. Myt1 family recruits histone deacetylase to regulate neural transcription. J. Neurochem. 93 (6), 1444-1453.

Strausberg, R.L., Feingold, E.A., Grouse, L.H., Derge, J.G., Klausner, R.D., Collins, F.S Wagner, L., Shenmen, C.M., Schuler, G.D., Altschul, S.F., Zeeberg, B., Buetow, K.H., Schaefer, C.F., Bhat, N.K., Hopkins, R.F., Jordan, H., Moore, T., Max, S.I., Wang, J.,
Hsieh, F., Diatchenko, L., Marusina, K., Farmer, A.A., Rubin, G.M., Hong, L., Stapleton, M., Soares, M.B., Bonaldo, M.F., Casavant, T.L., Scheetz, T.E., Brownstein, M.J., Usdin, T.B., Toshiyuki, S., Carninci, P., Prange, C., Raha, S.S., Loquellano, N.A., Peters, G.J., Abramson, R.D., Mullahy, S.J., Bosak, S.A., McEwan, P.J., McKernan, K.J., Malek, J.A., Gunaratne, P.H., Richards, S., Worley, K.C., Hale, S., Garcia, A.M., Gay, L.J., Hulyk, S.W., Villalon, D.K., Muzny, D.M., Sodergren, E.J., Lu, X., Gibbs, R.A., Fahey, J., Helton, E. Ketteman, M., Madan, A., Rodrigues, S., Sanchez, A., Whiting, M., Madan, A., Young, A.C., Shevchenko, Y., Bouffard, G.G., Blakesley, R.W., Touchman, J.W., Green, E.D., Dickson, M.C., Rodriguez, A.C., Grimwood, J., Schmutz, J., Myers, R.M., Butterfield, Y.S., Krzywinski, M.I., Skalska, U., Smailus, D.E., Schnerch, A., Schein, J.E., Jones, S.J., Marra, M.A., 2003. Generation and initial analysis of more than 15,000 full-length human and mouse cDNA sequences. Proc. Natl. Acad. Sci. U. S. A. 99 (26), 16899-16903.

Thomas, M.J., Kalivas, P.W., Shaham, Y., 2008. Neuroplasticity in the mesolimbic dopamine system and cocaine addiction. Br. J. Pharmacol. 154 (2), 327-342.

Tsankova, N., Renthal, W., Kumar, A., Nestler, E.J., 2007. Epigenetic regulation in psychiatric disorders. Nat. Rev., Neurosci. 8 (5), 355-367.

Trifunovski, A., Josephson, A., Ringman, A., Brené, S., Spenger, C., Olson, L., 2004. Neuronal activity-induced regulation of Lingo-1. NeuroReport 15 (15), 2397-2400.

Vanderschuren, L.J., Kalivas, P.W., 2000. Alterations in dopaminergic and glutamatergic transmission in the induction and expression of behavioral sensitization: a critical review of preclinical studies. Psychopharmacology (Berl) 151 (2-3), 99-120. 